\title{
SOLUTION OF A TRANSMISSION PROBLEM FOR SEMILINEAR PARABOLIC-HYPERBOLIC EQUATIONS BY THE TIME-DISCRETIZATION METHOD
}

\author{
ABDELFATAH BOUZIANI
}

Received 7 August 2004; Revised 7 May 2005; Accepted 24 May 2005

We consider a transmission problem for semilinear parabolic-hyperbolic equations. Existence, uniqueness, and continuous dependence of the solution upon the data are proved by using the time-discretization method. Besides, some convergence results of the approximations are established.

Copyright (C) 2006 Abdelfatah Bouziani. This is an open access article distributed under the Creative Commons Attribution License, which permits unrestricted use, distribution, and reproduction in any medium, provided the original work is properly cited.

\section{Introduction}

In the present paper, we consider a transmission problem for semilinear parabolichyperbolic equations in a multidimensional structure. Precisely, let $\Omega=\Omega_{1} \cup \Omega_{2}$ be an open bounded domain of $\mathbb{R}^{n}$ with sufficiently smooth boundary $\Gamma=\Gamma_{1} \cup \Gamma_{2}, \Omega_{1}$ and $\Omega_{2}$ having a common portion of boundary $\Sigma$. In the cylinder $Q=\Omega \times(0, T)$, let the unknown function $w=\left(w^{1}, w^{2}\right)$ :

$$
w(x, t)= \begin{cases}w^{1}(x, t), & (x, t) \in Q_{1}=\Omega_{1} \times(0, T), \\ w^{2}(x, t), & (x, t) \in Q_{2}=\Omega_{2} \times(0, T),\end{cases}
$$

checking the following couple of partial differential equations:

$$
\begin{array}{cc}
\frac{\partial w^{1}}{\partial t}-\Delta w^{1}=f^{1}\left(x, t, w^{1}\right), & \text { in } Q_{1}, \\
\frac{\partial^{2} w^{2}}{\partial t^{2}}-\Delta w^{2}=f^{2}\left(x, t, w^{2}\right), & \text { in } Q_{2},
\end{array}
$$

subject to the initial conditions

$$
\begin{gathered}
w(x, 0)=\left(w^{1}(x, 0), w^{2}(x, 0)\right)=\left(\varphi^{1}(x), \varphi^{2}(x)\right), \quad x \in \Omega, \\
\frac{\partial w^{2}(x, 0)}{\partial t}=\psi(x), \quad x \in \Omega_{2},
\end{gathered}
$$

Hindawi Publishing Corporation

Journal of Applied Mathematics and Stochastic Analysis

Volume 2006, Article ID 61439, Pages 1-23

DOI 10.1155/JAMSA/2006/61439 
2 A transmission semilinear parabolic-hyperbolic problem

the boundary conditions

$$
w=\left(w^{1}, w^{2}\right)=0, \quad \text { on } \Gamma \times(0, T),
$$

and the transmission conditions

$$
\begin{gathered}
w^{1}=w^{2}, \quad \text { on } \Sigma \times(0, T), \\
\frac{\partial w^{1}}{\partial \vartheta_{1}}+\frac{\partial w^{2}}{\partial \vartheta_{2}}=0, \quad \text { on } \Sigma \times(0, T),
\end{gathered}
$$

where $f^{p} \varphi^{p},(p=1,2), \psi$ are known functions.

The linear case of problem (1.2)-(1.5) has been studied by several authors. We refer the reader, for instance, to $[1,4,8,9]$ and references cited therein. In [8] the authors proved the existence and uniqueness of the solution for a similar problem, by using Oleĭnik's method [10]. However, the author of [9] has employed the variational method to investigate a boundary value problem where the geometrical domain consists of one 3-dimensional part $\Omega_{1}$ and one 1-dimensional part $\Omega_{2}$, with a point $A$ common to the two boundaries. The model which is studied by the author consists in a parabolic (resp., hyperbolic) equation in $\Omega_{1}$ (resp., $\Omega_{2}$ ) with transmission conditions at $A$. The work in [4] (resp., [1]) shows the existence and the uniqueness of the weak (resp., strong) solution, of a transmission problem for parabolic-hyperbolic equations by using the energyintegral method. Lastly, one quotes $[5,6]$ in which the author has used the energy-integral method to investigate more general problems.

Differently to these works, in the present paper we prove the well-posedness of problem (1.2)-(1.5) via approximation by using the time-discretization method. So, the semilinear parabolic-hyperbolic problem is approximated by a recurrent system of elliptic problems, to be solved at each subsequent time point.

To this end, we introduce two unknown functions:

$$
u^{1}(x, t):=w^{1}(x, t), \quad u^{2}(x, t):=\frac{\partial w^{2}(x, t)}{\partial t}
$$

then

$$
w^{2}(x, t)=\mathfrak{J}_{t} u^{2}+\varphi^{2}(x),
$$

where

$$
\mathfrak{J}_{t} u^{2}:=\int_{0}^{t} u^{2}(\cdot, s) d s
$$


So, problem (1.2)-(1.5) is seen to be equivalent to the following problem: find the function $u=\left(u^{1}, u^{2}\right)$ verifying

$$
\begin{gathered}
\frac{\partial u^{1}}{\partial t}-\Delta u^{1}=f^{1}\left(x, t, u^{1}\right), \quad \text { in } Q_{1}, \\
\frac{\partial u^{2}}{\partial t}-\Delta \mathfrak{J}_{t} u^{2}=f^{2}\left(x, t, \mathfrak{I}_{t} u^{2}+\varphi^{2}\right)+\Delta \varphi^{2}, \quad \text { in } Q_{2}, \\
u(x, 0)=u_{0}(x)=\left(\varphi^{1}(x), \psi(x)\right), \quad x \in \Omega, \\
u=\left(u^{1}, u^{2}\right)=0, \quad \text { on } \Gamma \times(0, T), \\
u^{1}=u^{2}, \quad \text { on } \Sigma \times(0, T), \\
\frac{\partial u^{1}}{\partial \vartheta_{1}}+\frac{\partial \mathfrak{I}_{t} u^{2}}{\partial \vartheta_{2}}+\frac{\partial \varphi^{2}}{\partial \vartheta_{2}}=0, \quad \text { on } \Sigma \times(0, T) .
\end{gathered}
$$

Hence, instead of looking for the function $w=\left(w^{1}, w^{2}\right)$, we search for the function $u=$ $\left(u^{1}, u^{2}\right)$. The solution of problem (1.2)-(1.5) will be given by $w^{1}=u^{1}$ and $w^{2}=\mathfrak{J}_{t} u^{2}+\varphi^{2}$.

In order to solve problem (1.9)-(1.12) by the time-discretization method, we divide the interval $I$ into $n$ subintervals by points $t_{j}=j h_{n}, j=0, \ldots, n$, where $h_{n}:=T / n$ is a step time. Set, for $j=1, \ldots, n$,

$$
\begin{gathered}
u_{j}=\left(u_{j}^{1}, u_{j}^{2}\right), \quad \text { with } u_{j}^{p}:=u^{p}\left(t_{j}\right), \quad \delta u_{j}^{p}=\frac{u_{j}^{p}-u_{j-1}^{p}}{h_{n}} \quad(p=1,2), \\
f_{j}^{1}:=f^{1}\left(t_{j}, u_{j-1}^{1}\right), \quad f_{j}^{2}:=f^{2}\left(t_{j}, h_{n} \sum_{i=1}^{j-1} u_{j-1}^{2}+\varphi^{2}\right) .
\end{gathered}
$$

Then, we are conducted to solve successively, for $j=1, \ldots, n$, the following linearized problems.

Starting from

$$
u_{0}=\left(u_{0}^{1}, u_{0}^{2}\right)=\left(\varphi^{1}, \psi\right)
$$

find functions $u_{j}: \Omega \rightarrow \mathbb{R}$ such that

$$
\begin{gathered}
\delta u_{j}^{1}-\Delta u_{j}^{1}=f_{j}^{1}, \quad \text { in } \Omega_{1}, \\
\delta u_{j}^{2}-h_{n} \Delta \sum_{i=1}^{j} \Delta u_{j}^{2}=f_{j}^{2}+\Delta \varphi^{2}, \quad \text { in } \Omega_{2}, \\
u_{j}=\left(u_{j}^{1}, u_{j}^{2}\right)=0, \quad \text { on } \Gamma, \\
u_{j}^{1}=u_{j}^{2}, \quad \text { on } \Sigma, \\
\frac{\partial u_{j}^{1}}{\partial \vartheta_{1}}+h_{n} \sum_{i=1}^{j} \frac{\partial u_{j}^{2}}{\partial \vartheta_{2}}+\frac{\partial \varphi^{2}}{\partial \vartheta_{2}}=0, \quad \text { on } \Sigma .
\end{gathered}
$$




\section{A transmission semilinear parabolic-hyperbolic problem}

The plan of this paper is as follows. Section 2 is devoted to some preliminaries: notations, appropriate function spaces, and basic assumptions on the data are introduced in Section 2.1, while in Section 2.2 we give some auxiliary results needed throughout the paper. The variational formulation of the problem and the concept of the solution we are considering are given in Section 2.3. In Section 3, we establish some a priori estimates for the discretized problem. Then, we proceed with some convergence results and prove the existence of the solution in Section 4. Finally, we demonstrate the uniqueness and continuous dependence of the solution upon the data in Section 5.

\section{Preliminaries}

2.1. Notations and assumptions. First, we introduce some functions obtained from the approximates $u_{j}^{p}(p=1,2)$ by piecewise linear interpolation and piecewise constant with respect to the time, respectively:

$$
u^{(n)}=\left(u^{1(n)}, u^{2(n)}\right), \quad \bar{u}^{(n)}=\left(\bar{u}^{1(n)}, \bar{u}^{2(n)}\right)
$$

with

$$
\begin{gathered}
u^{p(n)}(t)=u_{j-1}^{p}+\delta u_{j}^{p}\left(t-t_{j-1}\right) \quad(p=1,2), t \in\left[t_{j-1}, t_{j}\right], j=1, \ldots, n, \\
\bar{u}^{p(n)}(t)= \begin{cases}u_{0}^{p}, & \text { for } t \in\left[-h_{n}, 0\right], \\
u_{j}^{p}, & \text { for } t \in\left(t_{j-1}, t_{j}\right],(p=1,2), j=1, \ldots, n,\end{cases} \\
U^{2}(t):=\mathfrak{J}_{t} u^{2}, \\
U^{2(n)}(t):=\mathfrak{J}_{t} \bar{u}^{2(n)}, \\
\bar{U}^{2(n)}(t)= \begin{cases}h_{n} u_{0}^{2}, & \text { for } t \in\left[-h_{n}, 0\right], \\
h_{n} \sum_{i=1}^{j} u_{i}^{2}, & \text { for } t \in\left(t_{j-1}, t_{j}\right], j=1, \ldots, n .\end{cases}
\end{gathered}
$$

Moreover, we use the notation

$$
\begin{aligned}
\tau_{h_{n}} \bar{u}^{1(n)}(x, t) & =\bar{u}^{1(n)}\left(x, t-h_{n}\right), \\
\tau_{h_{n}} \bar{U}^{2(n)}(x, t) & =\bar{U}^{2(n)}\left(x, t-h_{n}\right),
\end{aligned}
$$

then we write

$$
\bar{f}^{p(n)}(t, w):=f^{p}\left(t_{j}, w\right) \quad(p=1,2)
$$


thus, for $t \in\left(t_{j-1}, t_{j}\right], j=1, \ldots, n$, we have

$$
\begin{gathered}
\bar{f}^{1(n)}\left(t, \tau_{h_{n}} \bar{u}^{1(n)}\right):=f^{1}\left(t_{j}, u_{j-1}^{1}\right)=f_{j}^{1}, \\
\bar{f}^{2(n)}\left(t, \tau_{h_{n}} \bar{U}^{2(n)}+\varphi^{2}\right):=f^{2}\left(t_{j}, h_{n} \sum_{i=1}^{j-1} u_{j-1}^{2}+\varphi^{2}\right)=f_{j}^{2} .
\end{gathered}
$$

Let $H^{1}\left(\Omega_{p}\right)$ be the usual first-order Sobolev space on $\Omega_{p}$ with scalar product $(\cdot, \cdot)_{1, \Omega_{p}}$ and corresponding norm $\|\cdot\|_{1, \Omega_{p}}$, let $(\cdot, \cdot)_{0, \Omega_{p}}$ and $\|\cdot\|_{0, \Omega_{p}}$ be the scalar product and corresponding norm, respectively, in $L^{2}\left(\Omega_{p}\right)$, and let

$$
H\left(\Delta, \Omega_{p}\right):=\left\{v^{p} \in H^{1}\left(\Omega_{p}\right), \Delta v^{p} \in L^{2}\left(\Omega_{p}\right),(p=1,2)\right\}
$$

endowed with the norm

$$
\left\|v^{p}\right\|_{H\left(\Delta, \Omega_{p}\right)}:=\left(\left\|v^{p}\right\|_{H^{1}\left(\Omega_{p}\right)}^{2}+\left\|\Delta v^{p}\right\|_{0, \Omega_{p}}^{2}\right)^{1 / 2} .
$$

We will also make frequent use of the seminorm

$$
\left|v^{p}\right|_{1, \Omega_{p}}=\left\|\nabla v^{p}\right\|_{0, \Omega_{p}}=\left(\sum_{i=1}^{n}\left\|\frac{\partial v^{p}}{\partial x_{i}}\right\|_{0, \Omega_{p}}^{2}\right)^{1 / 2} .
$$

Since $\Omega_{p}$ is bounded, there exists a constant $C\left(\Omega_{p}\right)$ such that

$$
\forall v^{p} \in V^{p}, \quad\left\|v^{p}\right\|_{0, \Omega_{p}} \leqslant C\left(\Omega_{p}\right)\left|v^{p}\right|_{1, \Omega_{p}}
$$

(known as the Poincaré-Friedrichs inequality). Therefore, the seminorm $|\cdot|_{1, \Omega_{p}}$ is a norm over the space $V^{p}$, equivalent to the norm $\|\cdot\|_{1, \Omega_{p}}$.

Let $V$ be the space of functions defined by

$$
V:=\left\{v=\left(v^{1}, v^{2}\right) / v^{p} \in V^{p}(p=1,2),\left.v^{1}\right|_{\Sigma}=\left.v^{2}\right|_{\Sigma}\right\}
$$

where

$$
V^{p}:=\left\{v^{p} \in H^{1}\left(\Omega_{p}\right) / v^{p}=0 \text { on } \Gamma_{p}\right\} \quad(p=1,2)
$$

The space $V$ is equipped with the norm $\|\cdot\|_{1, \Omega_{p}}$, defined by

$$
\|v\|_{1, \Omega}^{2}=\sum_{p=1}^{2}\left\|v^{p}\right\|_{1, \Omega_{p}}^{2}
$$

We identify $v \in V$ with a function $v: \Omega \rightarrow \mathbb{R}$ for which $\left.v\right|_{\Omega_{p}}=v^{p},(p=1,2)$. Similarly, we introduce the product space $\mathbb{L}^{2}(\Omega)=L^{2}\left(\Omega_{1}\right) \times L^{2}\left(\Omega_{2}\right)$ equipped with the scalar product 
6 A transmission semilinear parabolic-hyperbolic problem

and the associated norm

$$
(u, v)_{0, \Omega}=\sum_{p=1}^{2}\left(u^{p}, v^{p}\right)_{0, \Omega_{p}}, \quad\|u\|_{0, \Omega}^{2}=\sum_{p=1}^{2}\left\|u^{p}\right\|_{0, \Omega_{p}}^{2},
$$

respectively.

Moreover, we will use the standard functional spaces $L^{2}(I, H), C(I, H), L^{\infty}(I, H)$, and $C^{0,1}(I, H)$, where $H$ is a Hilbert space. For their properties, we refer the reader, for instance, to [7].

Next we formulate the assumptions which are supposed to hold throughout the paper. Assumption 2.1. $f^{p}\left(t, u^{p}\right): I \times L^{2}\left(\Omega_{p}\right) \rightarrow L^{2}\left(\Omega_{p}\right)$ is bounded in $L^{2}\left(\Omega_{p}\right)$ and fulfills the Lipschitz condition

$$
\left\|f^{p}\left(t, u^{p}\right)-f^{p}\left(t^{\prime}, u^{p^{\prime}}\right)\right\|_{0, \Omega_{p}} \leqslant L\left(\left|t-t^{\prime}\right|+\left\|u^{p}-u^{p^{\prime}}\right\|_{0, \Omega_{p}}\right)
$$

for all $t, t^{\prime} \in I$, and $u^{p}, u^{p^{\prime}} \in L^{2}\left(\Omega_{p}\right)(p=1,2)$.

Assumption 2.2. $\varphi^{p} \in H\left(\Delta, \Omega_{p}\right)(p=1,2) ; \psi \in H\left(\Delta, \Omega_{2}\right)$.

Assumption 2.3. Compatibility conditions: $\varphi^{1}=\psi$, and $\partial \varphi^{1} / \partial \vartheta_{1}+h_{n}\left(\partial \psi / \partial \vartheta_{2}\right)+\partial \varphi^{2} / \partial \vartheta_{2}$ $=0$, on $\Sigma$.

2.2. Auxiliary results. The following results are used in this paper. We list them for convenience.

Lemma 2.4 (an analogue of Gronwall's lemma in continuous form [2]). Let $f_{i}(t)(i=1$, 2) be real continuous functions on the interval $(0, T), f_{3}(t) \geqslant 0$ nondecreasing function on $t$, and $C>0$. If the inequality

$$
\int_{0}^{t} f_{1}(s) d s+f_{2}(t) \leqslant f_{3}(t)+C \int_{0}^{t} f_{2}(s) d s
$$

fulfills, then the inequality

$$
\int_{0}^{t} f_{1}(s) d s+f_{2}(t) \leqslant f_{3}(t) e^{C t}
$$

holds for all $t \in(0, T)$.

Lemma 2.5 (Gronwall's lemma in discrete form [11]). Let $\left\{a_{i}\right\}$ be a sequence of real, nonnegative numbers, and $A, C$, and $h_{n}$ are positive constants.

(1) If the inequality

$$
a_{j} \leqslant A+C h_{n} \sum_{i=1}^{j-1} a_{i}
$$


takes place for all $j=1, \ldots, n$, then the estimate

$$
a_{i} \leqslant A e^{C(j-1) h_{n}}
$$

holds for all $j=1,2, \ldots, n$.

(2) If the inequality

$$
a_{j} \leqslant A+C h_{n} \sum_{i=1}^{j} a_{i}
$$

fulfills and $h<1 / C$, then

$$
a_{i} \leqslant \frac{A}{1-C h_{n}} e^{C(j-1) h_{n} /\left(1-C h_{n}\right)}
$$

takes place for all $j=1,2, \ldots, n$.

Lemma 2.6 [3]. Let $V, Y$ be reflexive Banach spaces and let the imbedding $V \hookrightarrow Y$ be compact. If the estimates

$$
\int_{I}\left\|\frac{d u^{(n)}(t)}{d t}\right\|_{Y}^{2} d t \leqslant C, \quad\left\|\bar{u}^{(n)}(t)\right\|_{V} \leqslant C, \quad \forall t \in I
$$

hold for all $n \geqslant n_{0}>0$, then there exist $u \in C(I, Y) \cap L^{\infty}(I, V)$ with $d u / d t \in L^{2}(I, Y)$ (u is differentiable a.e. $t \in I)$ and a subsequence $\left\{u^{\left(n_{k}\right)}\right\}$ of $\left\{u^{(n)}\right\}$ such that $u^{\left(n_{k}\right)} \rightarrow u$ in $C(I, Y)$, $u^{\left(n_{k}\right)}(t) \rightarrow u(t), \bar{u}^{\left(n_{k}\right)}(t) \rightarrow u(t)$ in $V$ for all $t \in I$ and $d u^{\left(n_{k}\right)}(t) / d t \rightarrow d u(t) / d t$ in $L^{2}(I, Y)$. Moreover, if $\left\|d u^{(n)}(t) / d t\right\|_{Y} \leqslant C$ for all $n \geqslant n_{0}>0$ and a.e. $t \in I$, then $d u / d t \in L^{\infty}(I, Y)$ and $u: I \rightarrow Y$ is Lipschitz continuous, that is,

$$
\left\|u(t)-u\left(t^{\prime}\right)\right\|_{Y} \leqslant C\left|t-t^{\prime}\right|, \quad \forall t, t^{\prime} \in I .
$$

Moreover, the following identity will be frequently employed:

$$
(u(t)-w(t), u(t))_{0, \Omega}=\frac{1}{2}\left(\|u(t)\|_{0, \Omega}^{2}+\|u(t)-w(t)\|_{0, \Omega}^{2}-\|w(t)\|_{0, \Omega}^{2}\right),
$$

for all $t \in I$.

2.3. Variational formulation. Taking the scalar product in $\mathbb{L}^{2}(\Omega)$ of $(1.9)$ and $v=\left(v^{1}\right.$, $\left.v^{2}\right)$, we have

$$
\begin{aligned}
& \left(\frac{\partial u(\cdot, t)}{\partial t}, v\right)_{0, \Omega}-\left(\Delta u^{1}(\cdot, t), v^{1}\right)_{0, \Omega_{1}}-\left(\Delta \mathfrak{J}_{t} u^{2}, v^{2}\right)_{0, \Omega_{2}} \\
& \quad=\left(f^{1}\left(\cdot, t, u^{1}\right), v^{1}\right)_{0, \Omega_{1}}+\left(f^{2}\left(\cdot, t, \mathfrak{J}_{t} u^{2}+\varphi^{2}\right)+\Delta \varphi^{2}, v^{2}\right)_{0, \Omega_{2}} .
\end{aligned}
$$


8 A transmission semilinear parabolic-hyperbolic problem

Carrying out some integration by parts and using (1.12), (2.14), and (2.15), we get

$$
\begin{aligned}
& \left(\frac{\partial u(\cdot, t)}{\partial t}, v\right)_{0, \Omega}+\left(\nabla u^{1}(\cdot, t), \nabla v^{1}\right)_{0, \Omega_{1}}+\left(\nabla \mathfrak{J}_{t} u^{2}, \nabla v^{2}\right)_{0, \Omega_{2}} \\
& \quad=\left(f^{1}\left(\cdot, t, u^{1}\right), v^{1}\right)_{0, \Omega_{1}}+\left(f^{2}\left(\cdot, t, \mathfrak{J}_{t} u^{2}+\varphi^{2}\right), v^{2}\right)_{0, \Omega_{2}}-\left(\nabla \varphi^{2}, \nabla v^{2}\right)_{0, \Omega_{2}} .
\end{aligned}
$$

Then, we look for a weak solution in the following sense.

Definition 2.7. The weak solution of problem (1.9)-(1.12), intended to be a function $u: I \rightarrow \mathbb{L}^{2}(\Omega)$ verifying the following.

(i) $u \in C^{0,1}\left(I, \mathbb{L}^{2}(\Omega)\right) \cap L^{\infty}(I, V)$ and $u^{2} \in L^{2}\left(I, V^{2}\right)$.

(ii) $\mathfrak{J}_{t} u^{2} \in C^{0,1}\left(I, L^{2}\left(\Omega_{2}\right)\right) \cap L^{\infty}\left(I, V^{2}\right)$.

(iii) $u$ has a strong derivative (a.e. in $I) d u / d t \in L^{\infty}\left(I, \mathbb{L}^{2}(\Omega)\right)$.

(iv) $u(0)=u_{0}=\left(u_{0}^{1}, u_{0}^{2}\right)=\left(\varphi^{1}, \psi\right)$ in $\mathbb{L}^{2}(\Omega)$.

(v) Identity (2.29) holds for all $v \in V$ and $t \in I$.

We remark that since $u \in C^{0,1}\left(I, \mathbb{L}^{2}(\Omega)\right) \subset C^{1}\left(I, \mathbb{L}^{2}(\Omega)\right)$, the condition (iv) makes sense, and according to (i), (ii), (iii) together with Assumption 2.1 each term in the integral identity (2.29) is well defined.

Then, for each $n \geqslant 1$, problem (1.9)-(1.12) may be approximated by the following time-discretized problems.

Starting from the initial conditions

$$
u_{0}=\left(u_{0}^{1}, u_{0}^{2}\right)=\left(\varphi^{1}, \psi\right), \quad \text { in } \Omega \text {, }
$$

find successively for $j=1, \ldots, n$, functions $u_{j}: \Omega \rightarrow \mathbb{R}$, verifying the integral identity

$$
\begin{aligned}
&\left(\delta u_{j}, v\right)_{0, \Omega}+\left(\nabla u_{j}^{1}, \nabla v^{1}\right)_{0, \Omega_{1}}+h_{n}\left(\nabla \sum_{i=1}^{j} u_{i}^{2}, \nabla v^{2}\right)_{0, \Omega_{2}} \\
&=\left(f_{j}^{1}, v^{1}\right)_{0, \Omega_{1}}+\left(f_{j}^{2}, v^{2}\right)_{0, \Omega_{2}}-\left(\nabla \varphi^{2}, \nabla v^{2}\right)_{0, \Omega_{2}}, \quad \forall v \in V,
\end{aligned}
$$

which may be rewritten as follows:

$$
\begin{aligned}
\frac{1}{h_{n}}\left(u_{j}, v\right)_{0, \Omega}+\left(\nabla u_{j}^{1}, \nabla v^{1}\right)_{0, \Omega_{1}}+h_{n}\left(\nabla u_{j}^{2}, \nabla v^{2}\right)_{0, \Omega_{2}} & \\
= & \frac{1}{h_{n}}\left(u_{j-1}, v\right)_{0, \Omega}+\left(f_{j}^{1}, v^{1}\right)_{0, \Omega_{1}}+\left(f_{j}^{2}, v^{2}\right)_{0, \Omega_{2}} \\
& -\left(\nabla \varphi^{2}, \nabla v^{2}\right)_{0, \Omega_{2}}-h_{n}\left(\nabla \sum_{i=1}^{j-1} u_{i}^{2}, \nabla v^{2}\right), \quad \forall v \in V,
\end{aligned}
$$

together with the boundary conditions

$$
u_{j}=\left(u_{j}^{1}, u_{j}^{2}\right)=0, \quad \text { on } \Gamma,
$$


and the transmission conditions

$$
\begin{gathered}
u_{j}^{1}=u_{j}^{2}, \quad \text { on } \Sigma, \\
\frac{\partial u_{j}^{1}}{\partial \vartheta_{1}}+h_{n} \sum_{i=1}^{j} \frac{\partial u_{i}^{2}}{\partial \vartheta_{2}}+\frac{\partial \varphi^{2}}{\partial \vartheta_{2}}=0, \quad \text { on } \Sigma .
\end{gathered}
$$

Our assumptions permit a successive application of the Lax-Milgram theorem to conclude that the set of linear elliptic transmission problems admits a unique solution $u_{j}=$ $\left(u_{j}^{1}, u_{j}^{2}\right)$, for $j=1, \ldots, n$.

By using notation from Section 2.1, identity (2.31) may be written in the form

$$
\begin{aligned}
\left(\frac{d u^{(n)}}{d t}\right. & (t), v)_{0, \Omega}+\left(\nabla \bar{u}^{1(n)}(t), \nabla v^{1}\right)_{0, \Omega_{1}}+\left(\nabla \bar{U}^{2(n)}(t), \nabla v^{2}\right)_{0, \Omega_{2}} \\
= & \left(\bar{f}^{1(n)}\left(t, \tau_{h_{n}} \bar{u}^{1(n)}\right), v^{1}\right)_{0, \Omega_{1}}+\left(\bar{f}^{2(n)}\left(t, \tau_{h_{n}} \bar{U}^{2(n)}+\varphi^{2}\right), v^{2}\right)_{0, \Omega_{2}} \\
& -\left(\nabla \varphi^{2}, \nabla v^{2}\right)_{0, \Omega_{2}}, \quad \forall v \in V .
\end{aligned}
$$

\section{A priori estimates for the discretized problem}

Now, we derive some a priori estimates.

Lemma 3.1. Let Assumptions 2.1-2.3 be fulfilled. Then the following estimates:

$$
\begin{gathered}
\|\left.\delta u_{j}\right|_{0, \Omega} \leqslant C_{1}, \\
\left\|u_{j}\right\|_{0, \Omega} \leqslant C_{2}, \\
\left|u_{j}^{2}\right|_{1, \Omega_{2}} \leqslant C_{1},
\end{gathered}
$$

hold for $j=1, \ldots, n$, where $C_{1}$ and $C_{2}$ are positive constants independent of $h_{n}$ and $j$.

Proof. Taking the difference of identity (2.31) and the same identity written for $j-1$, and setting $v=\delta u_{j}=\left(\delta u_{j}^{1}, \delta u_{j}^{2}\right)$, we have

$$
\begin{aligned}
\left(\delta u_{j}\right. & \left.-\delta u_{j-1}, \delta u_{j}\right)_{0, \Omega}+\left(\nabla u_{j}^{1}-\nabla u_{j-1}^{1}, \nabla \delta u_{j}^{1}\right)_{0, \Omega_{1}}+h_{n}\left(\nabla u_{j}^{2}, \nabla \delta u_{j}^{2}\right)_{0, \Omega_{2}} \\
= & \left(f_{j}^{1}-f_{j-1}^{1}, \delta u_{j}^{1}\right)_{0, \Omega_{1}}+\left(f_{j}^{2}-f_{j-1}^{2}, \delta u_{j}^{2}\right)_{0, \Omega_{2}},
\end{aligned}
$$

whence, in view of identity (2.27) and some rearrangement, we obtain

$$
\begin{gathered}
\left\|\delta u_{j}\right\|_{0, \Omega}^{2}+h_{n}^{2}\left\|\delta^{2} u_{j}\right\|_{0, \Omega}^{2}+2 h_{n}\left|\delta u_{j}^{1}\right|_{1, \Omega_{1}}^{2}+2\left|u_{j}^{2}\right|_{1, \Omega_{2}}^{2} \\
=2\left(f_{j}^{1}-f_{j-1}^{1}, \delta u_{j}^{1}\right)_{0, \Omega_{1}}+2\left(f_{j}^{2}-f_{j-1}^{2}, \delta u_{j}^{2}\right)_{0, \Omega_{2}} \\
+\left\|\delta u_{j-1}\right\|_{0, \Omega}^{2}+2\left(\nabla u_{j}^{2}, \nabla u_{j-1}^{2}\right)_{0, \Omega_{2}} .
\end{gathered}
$$


10 A transmission semilinear parabolic-hyperbolic problem

Owing to the Cauchy inequality, it comes

$$
\begin{aligned}
& \left\|\delta u_{j}\right\|_{0, \Omega}^{2}+h_{n}^{2}\left\|\delta^{2} u_{j}\right\|_{0, \Omega}^{2}+2 h_{n}\left|\delta u_{j}^{1}\right|_{1, \Omega_{1}}^{2}+\left|u_{j}^{2}\right|_{1, \Omega_{2}}^{2} \\
& \quad \leqslant \frac{1}{h_{n}}\left(\left\|f_{j}^{1}-f_{j-1}^{1}\right\|_{0, \Omega_{1}}^{2}+\left\|f_{j}^{2}-f_{j-1}^{2}\right\|_{0, \Omega_{2}}^{2}\right)+h_{n}\left\|\delta u_{j}\right\|_{0, \Omega_{2}}^{2}+\left\|\delta u_{j-1}\right\|_{0, \Omega}^{2}+\left|u_{j-1}^{2}\right|_{1, \Omega_{2}}^{2} .
\end{aligned}
$$

Neglecting the second and third terms in the left-hand side of the last inequality and summing up over $i=2, \ldots, j$, this yields

$$
\begin{aligned}
& \left\|\delta u_{j}\right\|_{0, \Omega}^{2}+\left|u_{j}^{2}\right|_{1, \Omega_{2}}^{2} \\
& \quad \leqslant\left\|\delta u_{1}\right\|_{0, \Omega}^{2}+\left|u_{1}^{2}\right|_{1, \Omega_{2}}^{2}+\frac{1}{h_{n}} \sum_{i=2}^{j}\left(\left\|f_{i}^{1}-f_{i-1}^{1}\right\|_{0, \Omega_{1}}^{2}+\left\|f_{i}^{2}-f_{i-1}^{2}\right\|_{0, \Omega_{2}}^{2}\right)+h_{n} \sum_{i=2}^{j}\left\|\delta u_{j}\right\|_{0, \Omega}^{2} .
\end{aligned}
$$

According to Assumption 2.1, we get

$$
\begin{aligned}
\left\|f_{i}^{1}-f_{i-1}^{1}\right\|_{0, \Omega_{1}} & =\left\|f^{1}\left(t_{i}, u_{i-1}^{1}\right)-f^{1}\left(t_{i-1}, u_{i-2}^{1}\right)\right\|_{0, \Omega_{1}} \\
& \leqslant L\left(h_{n}+\left\|u_{i-1}^{1}-u_{i-2}^{1}\right\|_{0, \Omega_{1}}\right)=L h_{n}\left(1+\left\|\delta u_{i-1}^{1}\right\|_{0, \Omega_{1}}\right), \\
\left\|f_{i}^{2}-f_{i-1}^{2}\right\|_{0, \Omega_{2}} & =\left\|f^{2}\left(t_{i}, h_{n} \sum_{j=1}^{i-1} u_{j}^{2}+\varphi^{2}\right)-f^{2}\left(t_{i-1}, h_{n} \sum_{j=1}^{i-2} u_{j}^{2}+\varphi^{2}\right)\right\|_{0, \Omega_{2}} \\
& \leqslant L h_{n}\left(1+\left\|u_{i-1}^{2}\right\|_{0, \Omega_{2}}\right) .
\end{aligned}
$$

Consequently, it follows that

$$
\begin{aligned}
& \sum_{i=2}^{j}\left(\left\|f_{i}^{1}-f_{i-1}^{1}\right\|_{0, \Omega_{1}}^{2}+\left\|f_{i}^{2}-f_{i-1}^{2}\right\|_{0, \Omega_{2}}^{2}\right) \\
& \quad \leqslant 4 L^{2} h_{n}^{2}(j-1)+2 L^{2} h_{n}^{2} \sum_{i=2}^{j}\left\|\delta u_{i-1}^{1}\right\|_{0, \Omega_{1}}^{2}+2 L^{2} h_{n}^{2} \sum_{i=2}^{j}\left\|u_{i-1}^{2}\right\|_{0, \Omega_{2}}^{2},
\end{aligned}
$$

in light of which (3.7) becomes

$$
\begin{aligned}
\left\|\delta u_{j}\right\|_{0, \Omega}^{2}+\left|u_{j}^{2}\right|_{1, \Omega_{2}}^{2} \leqslant & \left\|\delta u_{1}\right\|_{0, \Omega}^{2}+\left|u_{1}^{2}\right|_{1, \Omega_{2}}^{2}+4 L^{2} T \\
& +h_{n} \sum_{i=2}^{j}\left\|\delta u_{j}\right\|_{0, \Omega}^{2}+2 L^{2} h_{n} \sum_{i=1}^{j-1}\left\|\delta u_{i}^{1}\right\|_{0, \Omega_{1}}^{2}+2 L^{2} h_{n} \sum_{i=1}^{j-1}\left\|u_{i}^{2}\right\|_{0, \Omega_{2}}^{2} .
\end{aligned}
$$


This inequality can be rewritten in the following way:

$$
\begin{aligned}
\left\|\delta u_{j}\right\|_{0, \Omega}^{2}+\left|u_{j}^{2}\right|_{1, \Omega_{2}}^{2} \leqslant & \left\|\delta u_{1}\right\|_{0, \Omega}^{2}+\left|u_{1}^{2}\right|_{1, \Omega_{2}}^{2}+4 L^{2} T \\
& +\left(2 L^{2}+1\right) h_{n} \sum_{i=1}^{j}\left\|\delta u_{i}\right\|_{0, \Omega}^{2}+2 L^{2} h_{n} \sum_{i=1}^{j-1}\left\|u_{i}^{2}\right\|_{0, \Omega_{2}}^{2} .
\end{aligned}
$$

Thus, owing to inequality (2.13), we get

$$
\begin{aligned}
\left\|\delta u_{j}\right\|_{0, \Omega}^{2}+\left|u_{j}^{2}\right|_{1, \Omega_{2}}^{2} \leqslant & \left\|\delta u_{1}\right\|_{0, \Omega}^{2}+\left|u_{1}^{2}\right|_{1, \Omega_{2}}^{2}+4 L^{2} T \\
& +\max \left(2 L^{2}+1,2 L^{2} C_{3}\right) h_{n} \sum_{i=1}^{j}\left(\left\|\delta u_{i}\right\|_{0, \Omega}^{2}+\left|u_{i}^{2}\right|_{1, \Omega_{2}}^{2}\right),
\end{aligned}
$$

where $C_{3}=C(\Omega)$. To estimate the first two terms in the right-hand side of (3.12), we consider identity (2.31) for $j=1$ :

$$
\begin{aligned}
& \left(\delta u_{1}, v\right)_{0, \Omega}+\left(\nabla u_{1}^{1}, \nabla v^{1}\right)_{0, \Omega_{1}}+h_{n}\left(\nabla u_{1}^{2}, \nabla v^{2}\right)_{0, \Omega_{2}} \\
& \quad=\left(f_{1}^{1}, v^{1}\right)_{0, \Omega_{1}}+\left(f_{1}^{2}, v^{2}\right)_{0, \Omega_{2}}-\left(\nabla \varphi^{2}, \nabla v^{2}\right)_{0, \Omega_{2}}, \quad \forall v \in V,
\end{aligned}
$$

hence

$$
\begin{aligned}
\left(\delta u_{1}, v\right)_{0, \Omega}+h_{n}\left(\nabla \delta u_{1}^{1}, \nabla v^{1}\right)_{0, \Omega_{1}}+h_{n}^{2}\left(\nabla \delta u_{1}^{2}, \nabla v^{2}\right)_{0, \Omega_{2}} & \\
= & \left(f_{1}^{1}, v^{1}\right)_{0, \Omega_{1}}+\left(f_{1}^{2}, v^{2}\right)_{0, \Omega_{2}}-\left(\nabla u_{0}^{1}, \nabla v^{1}\right)_{0, \Omega_{1}} \\
& -h_{n}\left(\nabla u_{0}^{2}, \nabla v^{2}\right)_{0, \Omega_{2}}-\left(\nabla \varphi^{2}, \nabla v^{2}\right)_{0, \Omega_{2}}, \quad \forall v \in V,
\end{aligned}
$$

so

$$
\begin{aligned}
\left(\delta u_{1}, v\right)_{0, \Omega}+h_{n}\left(\nabla \delta u_{1}^{1}, \nabla v^{1}\right)_{0, \Omega_{1}}+h_{n}^{2}\left(\nabla \delta u_{1}^{2}, \nabla v^{2}\right)_{0, \Omega_{2}} & \\
= & \left(f_{1}^{1}, v^{1}\right)_{0, \Omega_{1}}+\left(f_{1}^{2}, v^{2}\right)_{0, \Omega_{2}}-\left(\nabla \varphi^{1}, \nabla v^{1}\right)_{0, \Omega_{1}} \\
& \quad-h_{n}\left(\nabla \psi, \nabla v^{2}\right)_{0, \Omega_{2}}-\left(\nabla \varphi^{2}, \nabla v^{2}\right)_{0, \Omega_{2}}, \quad \forall v \in V .
\end{aligned}
$$

However, observe that

$$
\begin{aligned}
& -\left(\nabla \varphi^{1}, \nabla v^{1}\right)_{0, \Omega_{1}}=-\int_{\Gamma_{1} \cup \Sigma} \frac{\partial \varphi^{1}}{\partial \vartheta_{1}} v^{1} d \sigma+\left(\Delta \varphi^{1}, v^{1}\right)_{0, \Omega_{1}}, \\
& -h_{n}\left(\nabla \psi, \nabla v^{2}\right)_{0, \Omega_{2}}-\left(\nabla \varphi^{2}, \nabla v^{2}\right)_{0, \Omega_{2}} \\
& \quad=-\int_{\Gamma_{2} \cup \Sigma}\left(h_{n} \frac{\partial \psi}{\partial \vartheta_{2}}+\frac{\partial \varphi^{2}}{\partial \vartheta_{2}}\right) v^{2} d \sigma+h_{n}\left(\Delta \psi, v^{2}\right)_{0, \Omega_{2}}+\left(\Delta \varphi^{2}, v^{2}\right)_{0, \Omega_{2}} .
\end{aligned}
$$

Therefore, in view of (2.14), (2.15), and Assumption 2.3, we have

$$
\begin{gathered}
-\left(\nabla \varphi^{1}, \nabla v^{1}\right)_{0, \Omega_{1}}-h_{n}\left(\nabla \psi, \nabla v^{2}\right)_{0, \Omega_{2}}-\left(\nabla \varphi^{2}, \nabla v^{2}\right)_{0, \Omega_{2}} \\
=\left(\Delta \varphi^{1}, v^{1}\right)_{0, \Omega_{1}}+h_{n}\left(\Delta \psi, v^{2}\right)_{0, \Omega_{2}}+\left(\Delta \varphi^{2}, v^{2}\right)_{0, \Omega_{2}} .
\end{gathered}
$$


12 A transmission semilinear parabolic-hyperbolic problem

Substituting (3.17) into (3.15) yields

$$
\begin{aligned}
&\left(\delta u_{1}, v\right)_{0, \Omega}+h_{n}\left(\nabla \delta u_{1}^{1}, \nabla v^{1}\right)_{0, \Omega_{1}}+h_{n}^{2}\left(\nabla \delta u_{1}^{2}, \nabla v^{2}\right)_{0, \Omega_{2}} \\
&=\left(f_{1}^{1}+\Delta \varphi^{1}, v^{1}\right)_{0, \Omega_{1}}+\left(f_{1}^{2}+h_{n} \Delta \psi+\Delta \varphi^{2}, v^{2}\right)_{0, \Omega_{2}}, \quad \forall v \in V .
\end{aligned}
$$

Testing the resulting identity with $v=\delta u_{1}=\left(\delta u_{1}^{1}, \delta u_{1}^{2}\right)$, we obtain

$$
\begin{aligned}
\left\|\delta u_{1}\right\|_{0, \Omega}^{2} & +h_{n}\left|\delta u_{1}^{1}\right|_{1, \Omega_{1}}^{2}+h_{n}^{2}\left|\delta u_{1}^{2}\right|_{1, \Omega_{2}}^{2} \\
\leqslant & \left(\left\|f_{1}^{1}\right\|_{0, \Omega_{1}}+\left\|\Delta \varphi^{1}\right\|_{0, \Omega_{1}}\right)\left\|\delta u_{1}^{1}\right\|_{0, \Omega_{1}} \\
& +\left(\left\|f_{1}^{2}\right\|_{0, \Omega_{2}}+h_{n}\|\Delta \psi\|_{0, \Omega_{2}}+\left\|\Delta \varphi^{2}\right\|_{0, \Omega_{2}}\right)\left\|\delta u_{1}^{2}\right\|_{0, \Omega_{2}} .
\end{aligned}
$$

It is easy to see that

$$
\begin{aligned}
\left\|f_{1}^{1}\right\|_{0, \Omega_{1}} & \leqslant\left\|f^{1}\left(t_{1}, \varphi^{1}\right)-f^{1}\left(t_{1}, 0\right)\right\|_{0, \Omega_{1}}+\left\|f^{1}\left(t_{1}, 0\right)\right\|_{0, \Omega_{1}} \leqslant L\left\|\varphi^{1}\right\|_{0, \Omega_{1}}+M \\
\left\|f_{1}^{2}\right\|_{0, \Omega_{2}} & \leqslant\left\|f^{2}\left(t_{1}, h_{n} \psi+\varphi^{2}\right)-f^{2}\left(t_{1}, 0\right)\right\|_{0, \Omega_{2}}+\left\|f^{2}\left(t_{1}, 0\right)\right\|_{0, \Omega_{2}} \\
& \leqslant L h_{n}\|\psi\|_{0, \Omega_{2}}+L\left\|\varphi^{2}\right\|_{0, \Omega_{2}}+M
\end{aligned}
$$

where $M=\max _{t \in I}\|f P(t, 0)\|_{0, \Omega p}$. Then, inserting (3.20) into (3.19), it follows that

$$
\begin{aligned}
\left\|\delta u_{1}\right\|_{0, \Omega}^{2}+h_{n}\left|\delta u_{1}^{1}\right|_{1, \Omega_{1}}^{2}+h_{n}^{2}\left|\delta u_{1}^{2}\right|_{1, \Omega_{2}}^{2} & \\
\leqslant & \left(L\left\|\varphi^{1}\right\|_{0, \Omega_{1}}+\left\|\Delta \varphi^{1}\right\|_{0, \Omega_{1}}+M\right)\left\|\delta u_{1}^{1}\right\|_{0, \Omega_{1}} \\
& \quad+\left(h_{n}\left(L\|\psi\|_{0, \Omega_{2}}+\|\Delta \psi\|_{0, \Omega_{2}}\right)+L\left\|\varphi^{2}\right\|_{0, \Omega_{2}}+\left\|\Delta \varphi^{2}\right\|_{0, \Omega_{2}}+M\right)\left\|\delta u_{1}^{2}\right\|_{0, \Omega_{2}},
\end{aligned}
$$

which implies

$$
\begin{aligned}
& \frac{1}{2}\left\|\delta u_{1}\right\|_{0, \Omega}^{2}+h_{n}\left|\delta u_{1}^{1}\right|_{1, \Omega_{1}}^{2}+h_{n}^{2}\left|\delta u_{1}^{2}\right|_{1, \Omega_{2}}^{2} \\
& \quad \leqslant\left(\max 5\left(1, L^{2}, T^{2}, L^{2} T^{2}\right) / 2\right)\left(\left\|\varphi^{1}\right\|_{H\left(\Delta, \Omega_{1}\right)}^{2}+\left\|\varphi^{2}\right\|_{H\left(\Delta, \Omega_{2}\right)}^{2}+\|\psi\|_{H\left(\Delta, \Omega_{2}\right)}^{2}\right)+4 M^{2} .
\end{aligned}
$$

Hence, omitting the last two terms in the left-hand side of (3.22) yields

$$
\left\|\delta u_{1}\right\|_{0, \Omega} \leqslant C_{5}
$$

where

$$
C_{5}=\sqrt{\max 5\left(1, L^{2}, T^{2}, L^{2} T^{2}\right)\left(\left\|\varphi^{1}\right\|_{H\left(\Delta, \Omega_{1}\right)}^{2}+\left\|\varphi^{2}\right\|_{H\left(\Delta, \Omega_{2}\right)}^{2}+\|\psi\|_{H\left(\Delta, \Omega_{2}\right)}^{2}\right)+8 M^{2}} .
$$

However, since

$$
u_{1}=h_{n} \delta u_{1}+u_{0}
$$


then

$$
\left\|u_{1}\right\|_{0, \Omega} \leqslant h_{n}\left\|\delta u_{1}\right\|_{0, \Omega}+\left\|u_{0}\right\|_{0, \Omega}
$$

from which

$$
\left\|u_{1}\right\|_{0, \Omega} \leqslant C_{6}
$$

owing to (3.23), where $C_{6}=T C_{5}+\left\|u_{0}\right\|_{0, \Omega}$. On the other hand, consider identity (3.18), tested with $v=\left(v^{1}, v^{2}\right)=\left(u_{1}^{1}, \delta u_{1}^{2}\right)$, which is an element of $V$,

$$
\begin{gathered}
\left(\delta u_{1}^{1}, u_{1}^{1}\right)_{0, \Omega_{1}}+\left\|\delta u_{1}^{2}\right\|_{0, \Omega_{2}}^{2}+h_{n}\left(\nabla \delta u_{1}^{1}, \nabla u_{1}^{1}\right)_{0, \Omega_{1}}+h_{n}^{2}\left|\delta u_{1}^{2}\right|_{1, \Omega_{2}}^{2} \\
=\left(f_{1}^{1}+\Delta \varphi^{1}, u_{1}^{1}\right)_{0, \Omega_{1}}+\left(f_{1}^{2}+h_{n} \Delta \psi+\Delta \varphi^{2}, \delta u_{1}^{2}\right)_{0, \Omega_{2}} .
\end{gathered}
$$

Applying identity (2.27) to the third term in the left-hand side, hence (3.28) becomes after some rearrangement

$$
\begin{aligned}
& \|\left.\delta u_{1}^{2}\right|_{0, \Omega_{2}} ^{2}+\frac{1}{2}\left|u_{1}^{1}\right|_{1, \Omega_{1}}^{2}+\left|u_{1}^{2}\right|_{1, \Omega_{2}}^{2} \\
& \quad \leqslant \frac{1}{2}\left|\varphi^{1}\right|_{1, \Omega_{1}}^{2}+|\psi|_{1, \Omega_{2}}^{2}+\left(-\delta u_{1}^{1}+f_{1}^{1}+\Delta \varphi^{1}, u_{1}^{1}\right)_{0, \Omega_{1}}+\left(f_{1}^{2}+h_{n} \Delta \psi+\Delta \varphi^{2}, \delta u_{1}^{2}\right)_{0, \Omega_{2}},
\end{aligned}
$$

from which it comes

$$
\left\|\delta u_{1}^{2}\right\|_{0, \Omega_{2}}^{2}+\left|u_{1}\right|_{1, \Omega}^{2} \leqslant C_{7}
$$

where

$$
C_{7}=\max \left(3,3 L^{2}, 3 L^{2} T, T\right)\left(\left\|\varphi^{1}\right\|_{H\left(\Delta, \Omega_{1}\right)}^{2}+\left\|\varphi^{2}\right\|_{H\left(\Delta, \Omega_{2}\right)}^{2}+\|\psi\|_{H\left(\Delta, \Omega_{2}\right)}^{2}\right)+5 M^{2}+3 C_{6}^{2}+C_{5}^{2} .
$$

Consequently,

$$
\left|u_{1}^{2}\right|_{1, \Omega_{2}}^{2} \leqslant C_{7}
$$

Inserting (3.23) and (3.32) into (3.12) yields

$$
\left\|\delta u_{j}\right\|_{0, \Omega}^{2}+\left|u_{j}^{2}\right|_{1, \Omega_{2}}^{2} \leqslant C_{8}+\max \left(2 L^{2}+1,2 L^{2} C_{3}\right) h_{n} \sum_{i=1}^{j-1}\left(\left\|\delta u_{i}\right\|_{0, \Omega}^{2}+\left|u_{i}^{2}\right|_{1, \Omega_{2}}^{2}\right),
$$

with $C_{8}:=C_{5}^{2}+C_{7}$.

Thanks to Lemma 2.5, we obtain

$$
\left\|\delta u_{j}\right\|_{0, \Omega}^{2}+\left|u_{j}^{2}\right|_{1, \Omega_{2}}^{2} \leqslant C_{8} \exp \left(\max \left(2 L^{2}+1,2 L^{2} C_{3}\right)(j-1) h_{n}\right) \leqslant C_{9}, \quad \forall j=1,2, \ldots, n,
$$


14 A transmission semilinear parabolic-hyperbolic problem

where

$$
C_{9}=C_{8} \exp \left(\max \left(2 L^{2}+1,2 L^{2} C_{3}\right) T\right)
$$

from which we get estimates (3.1) and (3.3), with $C_{1}=C_{9}^{1 / 2}$. However, since

$$
u_{j}=h_{n} \sum_{i=1}^{j} \delta u_{j}+u_{0}
$$

then

$$
\left\|u_{j}\right\|_{0, \Omega} \leqslant h_{n} \sum_{i=1}^{j}\left\|\delta u_{j}\right\|_{0, \Omega}+\left\|u_{0}\right\|_{0, \Omega}
$$

whence

$$
\left\|u_{j}\right\|_{0, \Omega} \leqslant j h_{n} C_{1}+\left\|u_{0}\right\|_{0, \Omega}
$$

from which we obtain estimate (3.2) with $C_{2}=C_{1} T+\left\|u_{0}\right\|_{0, \Omega}$. This achieves the proof of Lemma 3.1.

As a consequence of Lemma 3.1, we have the following results.

Corollary 3.2. The functions $u^{(n)}$ and $\bar{u}^{(n)}$ obey the estimates

$$
\begin{gathered}
\left\|u^{(n)}(t)\right\|_{1, \Omega} \leqslant C_{2}, \quad \forall t \in I, \\
\left\|\bar{u}^{(n)}(t)\right\|_{1, \Omega} \leqslant C_{2}, \quad \forall t \in I, \\
\int_{I}\left\|\bar{u}^{2(n)}(t)\right\|_{0, \Omega_{2}}^{2} d t \leqslant C_{2}^{2} T, \quad \forall t \in I, \\
\left|\bar{U}^{2(n)}(t)\right|_{1, \Omega_{2}} \leqslant C_{1} T, \quad \forall t \in I, \\
\left\|\frac{d u^{(n)}(t)}{d t}\right\|_{0, \Omega} \leqslant C_{1}, \quad \text { a.e. in } I, \\
\left\|\bar{u}^{p(n)}(t)-\mathcal{u}^{p(n)}(t)\right\|_{0, \Omega_{p}} \leqslant C_{1} h_{n}, \quad \forall t \in I(p=1,2), \\
\left\|\bar{u}^{p(n)}(t)-\tau_{h_{n}} \bar{u}^{p(n)}(t)\right\|_{0, \Omega_{p}} \leqslant C_{1} h_{n}, \quad \forall t \in I(p=1,2), \\
\left\|\bar{U}^{2(n)}(t)-U^{2(n)}(t)\right\|_{0, \Omega_{2}} \leqslant C_{2} h_{n}, \quad \forall t \in I, \\
\left\|\bar{U}^{2(n)}(t)-\tau_{h_{n}} \bar{U}^{2(n)}(t)\right\|_{0, \Omega_{2}} \leqslant C_{2} h_{n}, \quad \forall t \in I,
\end{gathered}
$$

where $C_{10}=C_{1} T, C_{1}$ and $C_{2}$ are the same constants given in Lemma 3.1.

Proof. Estimates (3.39) are an immediate consequence of (3.1), with the same constant, while estimate (3.40a) follows directly from (3.39b). As for estimate (3.40b), we have, in 
light of (2.6)

$$
\bar{U}^{2(n)}=h_{n} \sum_{i=1}^{j} u_{i}^{2}, \quad \forall t \in\left(t_{j-1}, t_{j}\right], j=1, \ldots, n,
$$

so, according to (3.3), we have

$$
\left|\bar{U}^{2(n)}\right|_{1, \Omega_{2}} \leqslant h_{n} \sum_{i=1}^{j}\left|u_{i}^{2}\right|_{1, \Omega_{2}} \leqslant C_{1} h_{n} j \leqslant C_{10} .
$$

However, it follows from (2.2) that

$$
\frac{d u^{(n)}(t)}{d t}=\left(\frac{d u^{1(n)}(t)}{d t}, \frac{d u^{2(n)}(t)}{d t}\right)=\left(\delta u_{j}^{1}, \delta u_{j}^{2}\right)=\delta u_{j},
$$

for all, $t \in\left(t_{j-1}, t_{j}\right] j=1, \ldots, n$. Hence, thanks to (3.1), it is easy to get

$$
\left\|\frac{d u^{(n)}(t)}{d t}\right\|_{0, \Omega} \leqslant C_{1}, \quad \text { for a.e. } t \in I
$$

from which we have

$$
\int_{I}\left\|\frac{d u^{(n)}(t)}{d t}\right\|_{0, \Omega}^{2} d t \leqslant C_{11}
$$

with $C_{11}=C_{1}^{2} T$.

Next, observe that

$$
\begin{gathered}
\bar{u}^{p(n)}(t)-u^{p(n)}(t)=\left(t_{j}-t\right) \delta u_{j}^{p}, \quad \forall t \in\left(t_{j-1}, t_{j}\right](j=1, \ldots, n), \\
\bar{u}^{p(n)}(t)-\tau_{h_{n}} \bar{u}^{p(n)}(t)=u_{j}^{p}-u_{j-1}^{p}, \quad \forall t \in\left(t_{j-1}, t_{j}\right](j=1, \ldots, n),
\end{gathered}
$$

in view of (2.2)-(2.3), and (2.3), (2.7a), respectively. Consequently, we have

$$
\begin{aligned}
&\left\|\bar{u}^{p(n)}(t)-u^{p(n)}(t)\right\|_{0, \Omega_{p}} \leqslant C_{1} h_{n}, \forall t \in I, \\
&\left\|\bar{u}^{p(n)}(t)-\tau_{h_{n}} \bar{u}^{p(n)}(t)\right\|_{0, \Omega_{p}} \leqslant C_{1} h_{n}, \quad \forall t \in I,
\end{aligned}
$$

in view of (3.1). On the other hand, it comes from (2.5), (2.6), and (2.3) for $p=2$ :

$$
\begin{aligned}
& \bar{U}^{2(n)}(t)-U^{2(n)}(t)=h_{n} \sum_{i=1}^{j} u_{i}^{2}-\int_{0}^{t} \bar{u}^{2 n}(s) d s \\
& \quad=h_{n} \sum_{i=1}^{j} u_{i}^{2}-\sum_{i=1}^{j-1} \int_{(i-1) h_{n}}^{i h_{n}} \bar{u}^{2(n)}(s) d s-\int_{(i-1) h_{n}}^{t} \bar{u}^{2(n)}(s) d s=\left(t_{j}-t\right) u_{j}^{2}, \quad \forall t \in\left(t_{j-1}, t_{j}\right],
\end{aligned}
$$


16 A transmission semilinear parabolic-hyperbolic problem

therefore

$$
\left\|\bar{U}^{2(n)}(t)-U^{2(n)}(t)\right\|_{0, \Omega_{2}} \leqslant C_{2} h_{n}, \quad \forall t \in I,
$$

according to (3.2). Similarly, it follows from (2.6) and (2.7b), for $t \in\left(t_{j-1}, t_{j}\right]$,

$$
\bar{U}^{2(n)}(t)-\tau_{h_{n}} \bar{U}^{2(n)}(t)=h_{n} \sum_{i=1}^{j} u_{i}^{2}-h_{n} \sum_{i=1}^{j-1} u_{i}^{2}=h_{n} u_{j}^{2},
$$

so

$$
\left\|\bar{U}^{2(n)}(t)-\tau_{h_{n}} \bar{U}^{2(n)}(t)\right\|_{0, \Omega_{2}} \leqslant h_{n}\left\|u_{j}^{2}\right\|_{0, \Omega_{2}} \leqslant C_{2} h_{n},
$$

which completes the proof of Corollary 3.2 .

Moreover, we need two other estimates.

Lemma 3.3. Under Assumptions 2.1, the following estimates take place:

$$
\begin{aligned}
& \left|\left(\bar{f}^{1\left(n_{k}\right)}\left(t, \tau_{h_{n_{k}}} \bar{u}^{1\left(n_{k}\right)}\right), v^{1}\right)_{0, \Omega_{1}}+\left(\bar{f}^{2\left(n_{k}\right)}\left(t, \tau_{h_{n_{k}}} \bar{U}^{2\left(n_{k}\right)}+\varphi^{2}\right), v^{2}\right)_{0, \Omega_{2}}\right| \\
& \quad \leqslant C_{12}\|v\|_{0, \Omega}, \quad \forall v \in V, \forall t \in I, \\
& \left|\left(\nabla \bar{u}^{1\left(n_{k}\right)}(t), \nabla v^{1}\right)_{0, \Omega_{1}}+\left(\nabla \bar{U}^{2\left(n_{k}\right)}(t), \nabla v^{2}\right)_{0, \Omega_{2}}\right| \\
& \quad \leqslant C_{13}\|v\|_{1, \Omega}, \quad \forall v \in V \text {, a.e. } t \in I .
\end{aligned}
$$

Proof. Observe that

$$
\begin{aligned}
& \left|\left(\bar{f}^{1\left(n_{k}\right)}\left(t, \tau_{h_{n_{k}}} \bar{u}^{1\left(n_{k}\right)}\right), v^{1}\right)_{0, \Omega_{1}}+\left(\bar{f}^{2\left(n_{k}\right)}\left(t, \tau_{h_{n_{k}}} \bar{U}^{2\left(n_{k}\right)}+\varphi^{2}\right), v^{2}\right)_{0, \Omega_{2}}\right| \\
& \quad \leqslant\left\|\bar{f}^{1\left(n_{k}\right)}\left(t, \tau_{h_{n_{k}}} \bar{u}^{1\left(n_{k}\right)}\right)\right\|_{0, \Omega_{1}}\left\|v^{1}\right\|_{0, \Omega_{1}}+\left\|\bar{f}^{2\left(n_{k}\right)}\left(t, \tau_{h_{n_{k}}} \bar{U}^{2\left(n_{k}\right)}+\varphi^{2}\right)\right\|\left\|_{0, \Omega_{2}}\right\| v^{2} \|_{0, \Omega_{2}} \\
& \quad \leqslant\left\|f_{j}^{1}\right\|_{0, \Omega_{1}}\left\|v^{1}\right\|_{0, \Omega_{1}}+\left\|f_{j}^{2}\right\|_{0, \Omega_{2}}\left\|v^{2}\right\|_{0, \Omega_{2}},
\end{aligned}
$$

thanks to the Schwarz inequality and (2.9). By virtue of Assumption 2.1, we have

$$
\begin{aligned}
\left\|f_{j}^{1}\right\|_{0, \Omega_{1}} & \leqslant\left\|f^{1}\left(t_{j}, u_{j-1}^{1}\right)-f^{1}\left(t_{j}, 0\right)\right\|_{0, \Omega_{1}}+\left\|f^{1}\left(t_{j}, 0\right)\right\|_{0, \Omega_{1}} \\
& \leqslant L\left\|u_{j-1}^{1}\right\|_{0, \Omega_{1}}+M, \\
\left\|f_{j}^{2}\right\|_{0, \Omega_{2}} & \leqslant\left\|f^{2}\left(t_{j}, h_{n_{k}} \sum_{i=1}^{j-1} u_{i}^{2}+\varphi^{2}\right)-f^{2}\left(t_{j}, 0\right)\right\|_{0, \Omega_{2}}+\left\|f^{2}\left(t_{j}, 0\right)\right\|_{0, \Omega_{2}} \\
& \leqslant L h_{n_{k}} \sum_{i=1}^{j-1}\left\|u_{i}^{2}\right\|_{0, \Omega_{2}}+L\left\|\varphi^{2}\right\|_{0, \Omega_{2}}+M,
\end{aligned}
$$


from where, according to estimate (3.2), we obtain

$$
\begin{gathered}
\left\|f_{j}^{1}\right\|_{0, \Omega_{1}} \leqslant L C_{2}+M \\
\left\|f_{j}^{2}\right\|_{0, \Omega_{2}} \leqslant L\left(T C_{2}+\left\|\varphi^{2}\right\|_{0, \Omega_{2}}\right)+M
\end{gathered}
$$

Hence, substituting (3.59) into (3.57), we obtain (3.55), where $C_{12}=\sqrt{2} \max \left(L C_{2}+M\right.$, $\left.L\left(T C_{2}+\left\|\varphi^{2}\right\|_{0, \Omega_{2}}\right)+M\right)$.

On the other hand, it follows from the integral identity (2.35) that

$$
\begin{aligned}
\left|\left(\nabla \bar{u}^{1\left(n_{k}\right)}(t), \nabla v^{1}\right)_{0, \Omega_{1}}+\left(\nabla \bar{U}^{2\left(n_{k}\right)}(t), \nabla v^{2}\right)_{0, \Omega_{2}}\right| \\
=\left(\bar{f}^{1\left(n_{k}\right)}\left(t, \tau_{h_{n_{k}}} \bar{u}^{1\left(n_{k}\right)}\right), v\right)_{0, \Omega_{1}}+\left(\bar{f}^{2\left(n_{k}\right)}\left(t, \tau_{h_{n_{k}}} \bar{U}^{2\left(n_{k}\right)}+\varphi^{2}\right), v^{2}\right)_{0, \Omega_{2}} \\
\quad-\left(\frac{d u^{\left(n_{k}\right)}}{d t}(t), v\right)_{0, \Omega}-\left(\nabla \varphi^{2}, \nabla v^{2}\right)_{0, \Omega_{2}}, \quad \forall v \in V \text {, a.e. } t \in I,
\end{aligned}
$$

whence, owing to the Schwarz inequality and estimates (3.41) and (3.55), it comes that

$$
\begin{aligned}
& \left|\left(\nabla \bar{u}^{1\left(n_{k}\right)}(t), \nabla v^{1}\right)_{0, \Omega_{1}}+\left(\nabla \bar{U}^{2\left(n_{k}\right)}(t), \nabla v^{2}\right)_{0, \Omega_{2}}\right| \\
& \quad \leqslant\left(C_{12}+C_{1}\right)\|v\|_{0, \Omega}+\left|\varphi^{2}\right|_{1, \Omega_{2}}\left|v^{2}\right|_{1, \Omega_{2}}, \quad \forall v \in V \text {, a.e. } t \in I,
\end{aligned}
$$

from which we obtain (3.56), where $C_{13}=C_{12}+C_{1}+\left|\varphi^{2}\right|_{1, \Omega_{2}}$.

\section{Convergence and existence results}

First, owing to (3.39b), (3.41), and the continuous imbedding $V \hookrightarrow \mathbb{L}^{2}(\Omega)$, we obtain, on the basis of Lemma 2.6, that there exist

$$
u \in L^{\infty}(I, V) \cap C^{0,1}\left(I, \mathbb{L}^{2}(\Omega)\right),
$$

with

$$
\frac{d u}{d t} \in L^{\infty}\left(I, \mathbb{L}^{2}(\Omega)\right) \quad(u \text { is differentiable a.e. in } I),
$$

and subsequences $\left\{u^{\left(n_{k}\right)}\right\}$ and $\left\{\bar{u}^{\left(n_{k}\right)}\right\}$ of $\left\{u^{(n)}\right\}$ and $\left\{\bar{u}^{(n)}\right\}$, respectively, such that

$$
\begin{gathered}
u^{\left(n_{k}\right)} \longrightarrow u, \quad \text { in } C\left(I, \mathbb{L}^{2}(\Omega)\right), \\
u^{\left(n_{k}\right)}(t), \quad \bar{u}^{\left(n_{k}\right)}(t) \longrightarrow u(t), \quad \text { in } V \forall t \in I, \\
\frac{d u^{\left(n_{k}\right)}}{d t} \longrightarrow \frac{d u}{d t}, \quad \text { in } L^{2}\left(I, \mathbb{L}^{2}(\Omega)\right) .
\end{gathered}
$$


Similarly, by virtue of (3.40), and the continuous imbedding $V^{2} \hookrightarrow L^{2}\left(\Omega_{2}\right)$, we deduce that there exist

$$
\mathfrak{J}_{t} u^{2} \in L^{\infty}\left(I, V^{2}\right) \cap C^{0,1}\left(I, L^{2}\left(\Omega_{2}\right)\right),
$$

with

$$
u^{2} \in L^{\infty}\left(I, L^{2}\left(\Omega_{2}\right)\right)
$$

and subsequences $\left\{U^{2\left(n_{k}\right)}\right\}$ and $\left\{\bar{U}^{2\left(n_{k}\right)}\right\}$ of $\left\{U^{2(n)}\right\}$ and $\left\{\bar{U}^{2(n)}\right\}$, respectively, verifying

$$
\begin{gathered}
U^{2\left(n_{k}\right)} \longrightarrow U^{2}, \quad \text { in } C\left(I, L^{2}\left(\Omega_{2}\right)\right), \\
U^{2\left(n_{k}\right)}(t), \quad \bar{U}^{2\left(n_{k}\right)}(t) \longrightarrow U^{2}(t), \quad \text { in } V^{2} \forall t \in I, \\
\bar{u}^{2\left(n_{k}\right)} \longrightarrow u^{2}, \quad \text { in } L^{2}\left(I, V^{2}\right) .
\end{gathered}
$$

Therefore, the first three points of Definition 2.7 are already verified. Besides, since by definition $u^{\left(n_{k}\right)}(0)=\left(u^{1\left(n_{k}\right)}(0), u^{2\left(n_{k}\right)}(0)\right)=\left(u_{0}^{1}, u_{0}^{2}\right)=u_{0}$, it then follows from (4.3) that $u(0)=u_{0}$ holds in $\mathbb{L}^{2}(\Omega)$, thus point (iv) of Definition 2.7 is fulfilled. As for point $(\mathrm{v})$, we integrate identity (2.35) written for $n_{k}$, over $(0, t) \subset I$ by taking into account that if $u^{\left(n_{k}\right)}(0)=u_{0}$, we get

$$
\begin{aligned}
& \left(u^{\left(n_{k}\right)}(t)-u_{0}, v\right)_{0, \Omega}+\int_{0}^{t}\left(\left(\nabla \bar{u}^{1\left(n_{k}\right)}(s), \nabla v^{1}\right)_{0, \Omega_{1}}+\left(\nabla \bar{U}^{2\left(n_{k}\right)}(s), \nabla v^{2}\right)_{0, \Omega_{2}}\right) d s \\
& =\int_{0}^{t}\left(\left(\bar{f}^{1\left(n_{k}\right)}\left(s, \tau_{h_{n_{k}}} \bar{u}^{1\left(n_{k}\right)}\right), v^{1}\right)_{0, \Omega_{1}}+\left(\bar{f}^{2\left(n_{k}\right)}\left(s, \tau_{h_{n_{k}}} \bar{U}^{2\left(n_{k}\right)}+\varphi^{2}\right), v^{2}\right)_{0, \Omega_{2}}\right) d s \\
& \quad-t\left(\nabla \varphi^{2}, \nabla v^{2}\right)_{0, \Omega_{2}}, \quad \forall t \in I, \forall v \in V .
\end{aligned}
$$

In order to investigate the behavior of (4.11) as $n_{k}$ tends to infinity, we prove some convergence statements. First, due to (4.3), we have

$$
\left(u^{\left(n_{k}\right)}(t), v\right)_{0, \Omega} \underset{n_{k} \rightarrow \infty}{\longrightarrow}(u(t), v)_{0, \Omega}, \quad \forall t \in I .
$$

Furthermore, in view of (4.4) and (4.9), we deduce that

$$
\begin{gathered}
\left(\nabla \bar{u}^{1\left(n_{k}\right)}(t), \nabla v^{1}\right)_{0, \Omega_{1}}+\left(\nabla \bar{U}^{2\left(n_{k}\right)}(t), \nabla v^{2}\right)_{0, \Omega_{2}} \\
\underset{n_{k} \rightarrow \infty}{\longrightarrow}\left(\nabla u^{1}(t), \nabla v^{1}\right)_{0, \Omega_{1}}+\left(\nabla U^{2}(t), \nabla v^{2}\right)_{0, \Omega_{2}},
\end{gathered}
$$

from which together with estimate (3.56), we may apply the Lebesgue theorem of dominated convergence to obtain

$$
\begin{gathered}
\int_{0}^{t}\left(\left(\nabla \bar{u}^{1\left(n_{k}\right)}(s), \nabla v^{1}\right)_{0, \Omega_{1}}+\left(\nabla \bar{U}^{2\left(n_{k}\right)}(s), \nabla v^{2}\right)_{0, \Omega_{2}}\right) d s \\
\underset{n_{k} \rightarrow \infty}{\longrightarrow} \int_{0}^{t}\left(\left(\nabla u^{1}(s), \nabla v^{1}\right)_{0, \Omega_{1}}+\left(\nabla U^{2}(s), \nabla v^{2}\right)_{0, \Omega_{2}}\right) d s .
\end{gathered}
$$


On the other hand, by virtue of (2.9), Assumption 2.1, and estimates (3.42) at $p=1$, it follows, for all $t \in\left(t_{j-1}, t_{j}\right](j=1, \ldots, n)$, that

$$
\begin{aligned}
& \left\|\bar{f}^{1\left(n_{k}\right)}\left(t, \tau_{h_{n_{k}}} \bar{u}^{1\left(n_{k}\right)}\right)-f^{1}\left(t, u^{1}(t)\right)\right\|_{0, \Omega_{1}} \\
& =\left\|f^{1}\left(t_{j}, \tau_{h_{n_{k}}} \bar{u}^{1\left(n_{k}\right)}\right)-f^{1}\left(t, u^{1}(t)\right)\right\|_{0, \Omega_{1}} \\
& \leqslant L\left(\left|t_{j}-t\right|+\left\|\tau_{h_{n_{k}}} \bar{u}^{1\left(n_{k}\right)}-u^{1}(t)\right\|_{0, \Omega_{1}}\right) \\
& \leqslant L\left(h_{n_{k}}+\left\|\tau_{h_{h_{k}}} \bar{u}^{1\left(n_{k}\right)}-\bar{u}^{1\left(n_{k}\right)}(t)\right\|_{0, \Omega_{1}}+\left\|\bar{u}^{1\left(n_{k}\right)}(t)-u^{1\left(n_{k}\right)}(t)\right\|_{0, \Omega_{1}}\right. \\
& \left.\quad+\left\|u^{1\left(n_{k}\right)}(t)-u^{1}(t)\right\|_{0, \Omega_{1}}\right) \\
& \leqslant L\left(\left(1+2 C_{1}\right) h_{n_{k}}+\left\|u^{1\left(n_{k}\right)}-u^{1}\right\|_{C\left(I, L^{2}\left(\Omega_{1}\right)\right)}\right) .
\end{aligned}
$$

Hence, passing to the limit when $n_{k}$ tends to infinity by taking into account (4.3), we get

$$
\left\|\bar{f}^{1\left(n_{k}\right)}\left(t, \tau_{h_{n_{k}}} \bar{u}^{1\left(n_{k}\right)}\right)-f^{1}\left(t, u^{1}(t)\right)\right\|_{0, \Omega_{1}} \underset{n_{k} \rightarrow \infty}{\longrightarrow} 0, \quad \forall t \in I .
$$

Similarly, we have

$$
\begin{aligned}
& \left\|\bar{f}^{2\left(n_{k}\right)}\left(s, \tau_{h_{n_{k}}} \bar{U}^{2\left(n_{k}\right)}+\varphi^{2}\right)-f^{2}\left(s, U^{2}(t)+\varphi^{2}\right)\right\|_{0, \Omega_{2}} \\
& \leqslant L\left(h_{n_{k}}+\left\|\tau_{h_{n_{k}}} \bar{U}^{2\left(n_{k}\right)}-\bar{U}^{2\left(n_{k}\right)}(t)\right\|_{0, \Omega_{2}}+\left\|\bar{U}^{2\left(n_{k}\right)}(t)-U^{2\left(n_{k}\right)}(t)\right\|_{0, \Omega_{2}}\right. \\
& \left.\quad+\left\|U^{2\left(n_{k}\right)}(t)-U^{2}(t)\right\|_{0, \Omega_{2}}\right) \\
& \leqslant L\left(\left(1+2 C_{2}\right) h_{n_{k}}+\left\|U^{2\left(n_{k}\right)}(t)-U^{2}(t)\right\| \|_{C\left(I, L^{2}\left(\Omega_{2}\right)\right)}\right)
\end{aligned}
$$

in view of Assumption 2.1 and estimates (3.43). Then, owing to the continuous imbed$\operatorname{ding} V^{2} \hookrightarrow L^{2}\left(\Omega_{2}\right)$ and limit relation (4.8), we obtain

$$
\left\|\bar{f}^{2\left(n_{k}\right)}\left(t, \tau_{h_{n_{k}}} \bar{U}^{2\left(n_{k}\right)}+\varphi^{2}\right)-f^{2}\left(t, U^{2}(t)+\varphi^{2}\right)\right\|_{0, \Omega_{2}} \underset{n_{k} \rightarrow \infty}{\longrightarrow} 0, \quad \forall t \in I .
$$


Therefore, by virtue of estimates (3.55), and limit relations (4.16) and (4.18), the Lebesgue theorem of dominated convergence implies that

$$
\begin{gathered}
\int_{0}^{t}\left(\left(\bar{f}^{1\left(n_{k}\right)}\left(s, \tau_{h_{n_{k}}} \bar{u}^{1\left(n_{k}\right)}\right), v^{1}\right)_{0, \Omega_{1}}+\left(\bar{f}^{2\left(n_{k}\right)}\left(s, \tau_{h_{n_{k}}} \bar{U}^{2\left(n_{k}\right)}+\varphi^{2}\right), v^{2}\right)_{0, \Omega_{2}}\right) d s \\
\underset{n_{k} \rightarrow \infty}{\longrightarrow} \int_{0}^{t}\left(\left(f^{1}\left(s, u^{1}(s)\right), v^{1}\right)_{0, \Omega_{1}}+\left(f^{2}\left(s, \mathfrak{J}_{s} u^{2}+\varphi^{2}\right), v^{2}\right)_{0, \Omega_{2}}\right) d s,
\end{gathered}
$$

for all $v \in V$ and all $t \in I$.

Now we are ready to pass to the limit as $n_{k} \rightarrow \infty$ in (4.11). Observing (4.12), (4.14), and (4.19), then (4.11) passes into the following integral identity:

$$
\begin{aligned}
& (u(t), v)_{0, \Omega}+\int_{0}^{t}\left(\nabla u^{1}(s), \nabla v^{1}\right)_{0, \Omega_{1}} d s+\int_{0}^{t}\left(\nabla U^{2}(s), \nabla v^{2}\right)_{0, \Omega_{2}} d s \\
& =\int_{0}^{t}\left(\left(f^{1}\left(s, u^{1}(s)\right), v^{1}\right)_{0, \Omega_{1}}+\left(f^{2}\left(s, \mathfrak{J}_{s} u^{2}+\varphi^{2}\right), v^{2}\right)_{0, \Omega_{2}}\right) d s \\
& \quad+\left(u_{0}, v\right)_{0, \Omega}-t\left(\nabla \varphi^{2}, \nabla v^{2}\right)_{0, \Omega_{2}},
\end{aligned}
$$

for all $t \in I$, for all $v \in V$. Since $u: I \rightarrow V$ is strongly differentiable for a.e. $t \in I$, then the differentiation of the above identity with respect to $t$ leads to the desired identity (2.35) thanks to the equality

$$
\frac{d}{d t}(u(t), v)_{0, \Omega}=\left(\frac{d}{d t} u(t), v\right)_{0, \Omega}, \quad \text { a.e. } t \in I, \forall v \in V .
$$

Thus, we have proved the following theorem.

Theorem 4.1. Let Assumptions 2.1-2.3 be fulfilled. Then, problem (1.9)-(1.12) admits at most a solution in the sense of Definition 2.7, verifying $u \in L^{\infty}(I, V) \cap C^{0,1}\left(I, \mathbb{L}^{2}(\Omega)\right)$ with $u^{2} \in L^{2}\left(I, V^{2}\right), d u / d t \in L^{\infty}\left(I, \mathbb{L}^{2}(\Omega)\right)$, and $\mathfrak{J}_{t} u^{2} \in L^{\infty}\left(I, V^{2}\right) \cap C^{0,1}\left(I, L^{2}\left(\Omega_{2}\right)\right)$.

A subsequence $\left\{u^{\left(n_{k}\right)}\right\}\left[\left\{\bar{u}^{\left(n_{k}\right)}\right\}\right.$, resp.) of $\left\{u^{(n)}\right\}$ and $\left(\left\{\bar{u}^{(n)}\right\}\right.$, resp.) converges to the solution $u$ in the following sense:

(i) $u^{\left(n_{k}\right)} \rightarrow u$ in $C\left(I, \mathbb{L}^{2}(\Omega)\right)$;

(ii) $u^{\left(n_{k}\right)}(t) \rightarrow u(t)$ in $V$ for all $t \in I$;

(iii) $\bar{u}^{\left(n_{k}\right)}(t) \rightarrow u(t)$ in $V$ for all $t \in I$;

(iv) $U^{2\left(n_{k}\right)} \rightarrow \mathfrak{J}_{t} u^{2}$ in $C\left(I, L^{2}\left(\Omega_{2}\right)\right)$;

(v) $U^{2\left(n_{k}\right)}(t), \bar{U}^{2\left(n_{k}\right)}(t) \rightarrow \mathfrak{I}_{t} u^{2}$ in $V^{2}$ for all $t \in I$;

(vi) $\bar{u}^{2\left(n_{k}\right)}-u^{2}$ in $L^{2}\left(I, L^{2}\left(\Omega_{2}\right)\right)$;

(vii) $d u^{\left(n_{k}\right)} / d t \rightarrow d u / d t$ in $L^{2}\left(I, \mathbb{L}^{2}(\Omega)\right)$,

as $n_{k}$ tends to infinity.

\section{Continuous dependence and uniqueness}

We, first, prove the continuous dependence of the solution upon the data. Then, the uniqueness is an immediate corollary of it. To this end, we subtract the integral identity (2.29) written for $u^{* *}$ from (2.29) written for $u^{*}$, and setting $v=u^{*}(t)-u^{* *}(t)$ in 
the obtained identity, we find for a.e. $t \in I$,

$$
\begin{aligned}
\left(\frac{d}{d t}\left(u^{*}(t)-u^{* *}(t)\right), u^{*}(t)-u^{* *}(t)\right)_{0, \Omega} \\
\quad+\left(\nabla u^{1 *}(t)-\nabla u^{1 * *}(t), \nabla u^{1 *}(t)-\nabla u^{1 * *}(t)\right)_{0, \Omega_{1}} \\
\quad+\left(\nabla\left(\mathfrak{I}_{t} u^{2 *}+\varphi^{2 *}\right)-\nabla\left(\mathfrak{I}_{t} u^{2 * *}+\varphi^{2 * *}\right), \nabla u^{2 *}(t)-\nabla u^{2 * *}(t)\right)_{0, \Omega_{2}} \\
=\left(f^{1 *}\left(t, u^{1 *}(t)\right)-f^{1 * *}\left(t, u^{1 * *}(t)\right), u^{1 *}(t)-u^{1 * *}(t)\right)_{0, \Omega_{1}} \\
\quad+\left(f^{2 *}\left(t, \mathfrak{I}_{t} u^{2 *}+\varphi^{2 *}\right)-f^{2 * *}\left(t, \mathfrak{I}_{t} u^{2 * *}+\varphi^{2 * *}\right), u^{2 *}(t)-u^{2 * *}(t)\right)_{0, \Omega_{2}},
\end{aligned}
$$

whence

$$
\begin{aligned}
2 \mid u^{1 *}( & t)-\left.u^{1 * *}(t)\right|_{1, \Omega_{1}} ^{2}+\frac{d}{d t}\left\|u^{*}(t)-u^{* *}(t)\right\|_{0, \Omega}^{2}+\frac{d}{d t}\left|\left(\mathfrak{J}_{t} u^{2 *}+\varphi^{2 *}\right)-\left(\mathfrak{J}_{t} u^{2 * *}+\varphi^{2 * *}\right)\right|_{1, \Omega_{2}}^{2} \\
\leqslant & 2\left\|f^{1 *}\left(t, u^{1 *}(t)\right)-f^{1 * *}\left(t, u^{1 * *}(t)\right)\right\|_{0, \Omega_{1}}\left\|u^{1 *}(t)-u^{1 * *}(t)\right\|_{0, \Omega_{1}} \\
& +2\left\|f^{2 *}\left(t, \mathfrak{J}_{t} u^{* 2}+\varphi^{* 2}\right)-f^{2 * *}\left(t, \mathfrak{I}_{s} u^{* * 2}+\varphi^{* * 2}\right)\right\|_{0, \Omega_{2}} \times\left\|u^{2 *}(t)-u^{2 * *}(t)\right\|_{0, \Omega_{2}} .
\end{aligned}
$$

Then integrating (5.2) over $(0, t)$ and applying the Cauchy inequality, it follows that

$$
\begin{aligned}
& 2 \int_{0}^{t}\left|u^{1 *}(s)-u^{1 * *}(s)\right|_{1, \Omega_{1}}^{2} d s+\left\|u^{*}(t)-u^{* *}(t)\right\|_{0, \Omega}^{2}+\left|\left(\mathfrak{J}_{t} u^{2 *}+\varphi^{2 *}\right)-\left(\mathfrak{J}_{t} u^{2 * *}+\varphi^{2 * *}\right)\right|_{1, \Omega_{2}}^{2} \\
& \leqslant \int_{0}^{t}\left\|f^{1 *}\left(s, u^{1 *}(s)\right)-f^{1 * *}\left(s, u^{1 * *}(s)\right)\right\|_{0, \Omega_{1}}^{2} d s \\
&+\int_{0}^{t}\left\|f^{2 *}\left(s, \mathfrak{J}_{s} u^{* 2}+\varphi^{* 2}\right)-f^{2 * *}\left(s, \mathfrak{J}_{s} u^{* * 2}+\varphi^{* * 2}\right)\right\|_{0, \Omega_{2}}^{2} d s \\
&+\int_{0}^{t}\left\|u^{*}(s)-u^{* *}(s)\right\|_{0, \Omega}^{2} d s .
\end{aligned}
$$

According to Lemma 2.4, (5.3) becomes

$$
\begin{gathered}
\left\|u^{*}(t)-u^{* *}(t)\right\|_{0, \Omega}^{2}+2 \int_{0}^{t}\left|u^{1 *}(s)-u^{1 * *}(s)\right|_{1, \Omega_{1}}^{2} d s+\left|\left(\mathfrak{J}_{t} u^{2 *}+\varphi^{2 *}\right)-\left(\mathfrak{J}_{t} u^{2 * *}+\varphi^{2 * *}\right)\right|_{1, \Omega_{2}}^{2} \\
\leqslant e^{T} \int_{0}^{t}\left(\left\|f^{1 *}\left(s, u^{1 *}(s)\right)-f^{1 * *}\left(s, u^{1 * *}(s)\right)\right\|_{0, \Omega_{1}}^{2}\right. \\
\left.\quad+\left\|f^{2 *}\left(s, \mathfrak{J}_{s} u^{* 2}+\varphi^{* 2}\right)-f^{2 * *}\left(s, \mathfrak{J}_{s} u^{* * 2}+\varphi^{* * 2}\right)\right\|_{0, \Omega_{2}}^{2}\right) d s .
\end{gathered}
$$

Consequently, we can state the following theorem.

Theorem 5.1. Assume that $\left(f^{*}, u^{0 *}\right)$ and $\left(f^{* *}, u^{0 * *}\right)$ satisfy Assumptions 2.1-2.3. Let $u^{*}(x, t)$ and $u^{* *}(x, t)$ be two solutions of problem (1.9)-(1.12) corresponding, respectively, 
22 A transmission semilinear parabolic-hyperbolic problem

to the above data. Then the estimate

$$
\begin{aligned}
& \left\|u^{*}(t)-u^{* *}(t)\right\|_{0, \Omega}^{2} \\
& \qquad C\left(\int _ { 0 } ^ { t } \left(\left\|f^{1 *}\left(s, u^{1 *}(s)\right)-f^{1 * *}\left(s, u^{1}(s)\right)\right\|_{0, \Omega_{1}}+\| f^{2 *}\left(s, \mathfrak{I}_{s} u^{2 *}+\varphi^{2 *}\right)\right.\right. \\
& \left.\left.\quad-f^{2 * *}\left(s, \mathfrak{I}_{s} u^{2 * *}+\varphi^{2 * *}\right) \|_{0, \Omega_{2}}\right) d s+\left\|u^{0 *}-u^{0 * *}\right\|_{0, \Omega}^{2}\right)
\end{aligned}
$$

takes place for all $t \in I$, where $C$ is a positive constant depending only on the known data.

This theorem leads to the following result.

COROLlary 5.2. Under assumptions of Theorem 5.1, the weak solution of problem (1.9)(1.12) is unique.

Proof. Assume that $\hat{u}$ and $\tilde{u}$ are two weak solutions of (1.9)-(1.12). Taking the difference of the integral identities (2.29) corresponding to $\hat{u}$ and $\tilde{u}$ tested with $v=\hat{u}-\tilde{u}$ and performing a similar calculation to that for Theorem 5.1, we obtain

$$
\begin{aligned}
& 2 \int_{0}^{t}\left|\hat{u}^{1}(s)-\tilde{u}^{1}(s)\right|_{1, \Omega_{1}}^{2} d s+\|\hat{u}(t)-\tilde{u}(t)\|_{0, \Omega}^{2}+\left|\mathfrak{I}_{t} \hat{u}^{2}-\mathfrak{I}_{t} \tilde{u}^{2}\right|_{1, \Omega_{2}}^{2} \\
& \leqslant \int_{0}^{t}\left\|f^{1}\left(s, \hat{u}^{1}(s)\right)-f^{1}\left(s, \tilde{u}^{1}(s)\right)\right\|_{0, \Omega_{1}}^{2} d s \\
&+\int_{0}^{t}\left\|f^{2}\left(s, \mathfrak{J}_{s} \hat{u}^{2}+\varphi^{2}\right)-f^{2}\left(s, \mathfrak{J}_{s} \tilde{u}^{2}+\varphi^{2}\right)\right\|_{0, \Omega_{2}}^{2} d s \\
&+\int_{0}^{t}\|\hat{u}(s)-\tilde{u}(s)\|_{0, \Omega}^{2} d s .
\end{aligned}
$$

Employing Assumption 2.1 and omitting the first term in the left-hand side of (5.6), we find

$$
\begin{aligned}
& \|\hat{u}(t)-\tilde{u}(t)\|_{0, \Omega}^{2}+\left|\mathfrak{J}_{t} \hat{\mathcal{u}}^{2}-\mathfrak{J}_{t} \tilde{u}^{2}\right|_{1, \Omega_{2}}^{2} \\
& \quad \leqslant L^{2} \int_{0}^{t}\left\|\hat{u}^{1}(s)-\tilde{u}^{1}(s)\right\|_{0, \Omega_{1}}^{2} d s+\int_{0}^{t}\left\|\mathfrak{J}_{s} \hat{\mathcal{u}}^{2}-\mathfrak{I}_{s} \tilde{u}^{2}\right\|_{0, \Omega_{2}}^{2} d s+\int_{0}^{t}\|\hat{u}(s)-\tilde{u}(s)\|_{0, \Omega}^{2} d s,
\end{aligned}
$$

from which, by using inequality (2.13), we get

$$
\begin{aligned}
& \|\hat{u}(t)-\tilde{u}(t)\|_{0, \Omega}^{2}+\left|\mathfrak{J}_{t} \hat{u}^{2}-\mathfrak{J}_{t} \tilde{u}^{2}\right|_{1, \Omega_{2}}^{2} \\
& \quad \leqslant \max \left(L^{2}+1, C(\Omega)\right)\left(\int_{0}^{t}\|\hat{u}(s)-\tilde{u}(s)\|_{0, \Omega}^{2} d s+\int_{0}^{t}\left|\mathfrak{J}_{s} \hat{u}^{2}-\mathfrak{J}_{s} \tilde{u}^{2}\right|_{1, \Omega_{2}}^{2} d s\right) .
\end{aligned}
$$

Thanks to Lemma 2.4, we deduce that $\hat{u}=\tilde{u}$, which completes the proof.

Remark 5.3. By standard arguments we see that the uniqueness of the solution implies that all our convergence results for subsequences (4.3)-(4.10) take place for the whole sequences corresponding to a subdivision with arbitrary $h_{n} \rightarrow 0$, too. 
Remark 5.4. Our results can be extended to the following differential equations:

$$
\begin{aligned}
& \frac{\partial w^{1}}{\partial t}+A^{(1)} w^{1}+S^{(1)} w^{1}=f^{1}\left(x, t, w^{1}\right), \quad \text { in } Q_{1}, \\
& \frac{\partial^{2} w^{2}}{\partial t^{2}}+A^{(2)} w^{2}+S^{(2)} w^{2}=f^{2}\left(x, t, w^{2}\right), \quad \text { in } Q_{2},
\end{aligned}
$$

with corresponding boundary and transmission conditions $[5,6]$, where $A^{(p)}$ $=\sum_{|\alpha| \leqslant 2 m} a_{\alpha}^{(p)}(x) D^{\alpha}$ is a positive elliptic operator in $\overline{\Omega_{p}}$ for each $t \in I, S^{(p)}=\partial^{p} / \partial t^{p}+$ $\varrho(x) \partial^{p-1} / \partial t^{p-1}+\sum_{|\mu| \leqslant m} \sigma_{\mu}^{(p)}(x) D^{\mu}(p=1,2)$. Here $D=\left(D_{1}, \ldots, D_{n}\right), D_{k}=-i \partial / \partial x_{k}, \alpha=$ $\left(\alpha_{1}, \ldots, \alpha_{n}\right), \mu=\left(\mu_{1}, \ldots, \mu_{n}\right),|\alpha|=\left|\alpha_{1}\right|+\cdots+\left|\alpha_{n}\right|$, and $|\mu|=\left|\mu_{1}\right|+\cdots+\left|\mu_{n}\right|$.

\section{References}

[1] A. Bouziani, Solution forte d'un problème de transmission parabolique-hyperbolique pour une structure pluridimensionnelle [Strong solution of a parabolic-hyperbolic jump problem for a multidimensional structure], Académie Royale de Belgique. Bulletin de la Classe des Sciences. 6e Série 7 (1996), no. 7-12, 369-386 (1997) (French).

[2] L. Gårding, Cauchy's Problem For Hyperbolic Equations, Lecture Notes, University of Chicago, Chicago, 1957.

[3] J. Kačur, Method of Rothe in Evolution Equations, Teubner-Texte zur Mathematik, vol. 80, BSB B. G. Teubner Verlagsgesellschaft, Leipzig, 1985.

[4] V. I. Korzjuk, A conjugacy problem for equations of hyperbolic and parabolic types, Differentsial' nye Uravneniya 4 (1968), no. 10, 1854-1866 (Russian).

[5] V. I. Korzjuk, A mixed problem for certain nonstationary equations with discontinuous coefficients, Differentsial' nye Uravneniya 6 (1970), no. 2, 343-357 (Russian).

[6] The problem of the conjugacy of certain nonstationary differential equations of order $2 \mathrm{~m}$, Differentsial' nye Uravneniya 7 (1971), no. 4, 750-753 (Russian).

[7] A. Kufner, O. John, and S. Fučík, Function Spaces, Monographs and Textbooks on Mechanics of Solids and Fluids; Mechanics: Analysis, Noordhoff, Leyden; Academia, Prague, 1977.

[8] O. A. Ladyzhenskaya and L. I. Stupyalis, Problème de conjugaison de l'équation ondulatoire et de l'équation de conductibilité thermique, Differentsial' nye Uravneniya 1 (1965), no. 4, 38-46 (French).

[9] J.-L. Lions, Un exemple de problème aux limites couplé parabolique-hyperbolique pour une structure pluri-dimensionnelle [An example of a coupled parabolic-hyperbolic boundary value problem for a pluridimensional structure], Calcolo 22 (1985), no. 1, 7-15 (French).

[10] O. A. Olen̆nik, Boundary-value problems for linear equations of elliptic parabolic type with discontinuous coefficients, Izvestiya Akademii Nauk SSSR. Seriya Matematicheskaya 25 (1961), no. 1, 3-20 (Russian).

[11] K. Rektorys, The Method of Discretization in Time and Partial Differential Equations, Mathematics and Its Applications (East European Series), vol. 4, D. Reidel, Dordrecht, 1982.

Abdelfatah Bouziani: Department of Mathematics, The Larbi Ben M'hidi University Centre, P.O. Box 565, Oum El-Bougui 04000, Algeria

E-mail addresses: af_bouziani@hotmail.com; aefbouziani@yahoo.fr 


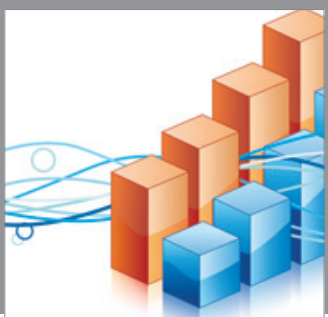

Advances in

Operations Research

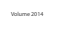

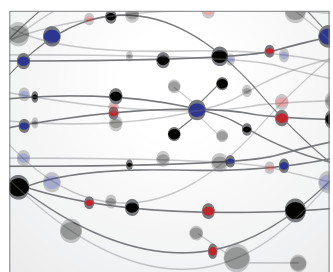

\section{The Scientific} World Journal
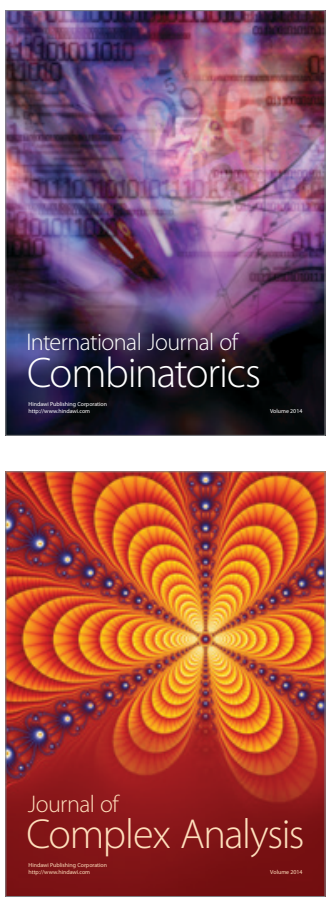

International Journal of

Mathematics and

Mathematical

Sciences
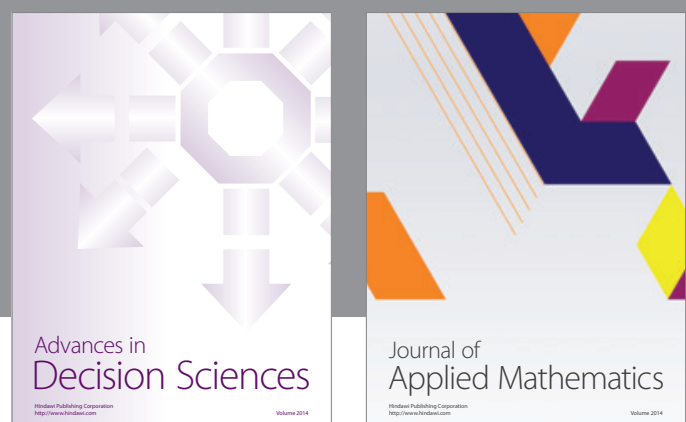

Journal of

Applied Mathematics
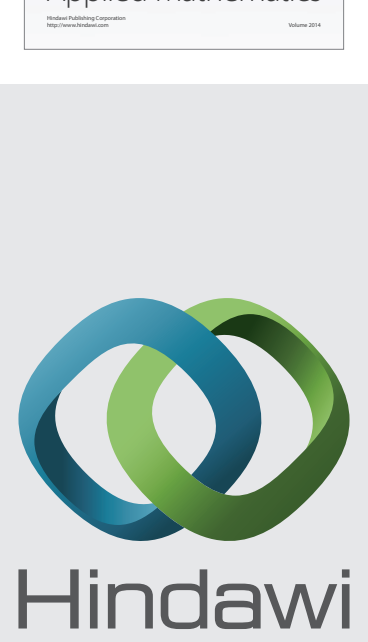

Submit your manuscripts at http://www.hindawi.com
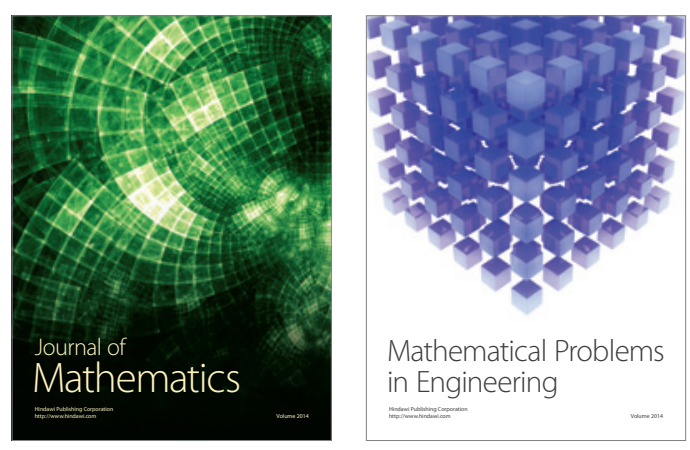

Mathematical Problems in Engineering
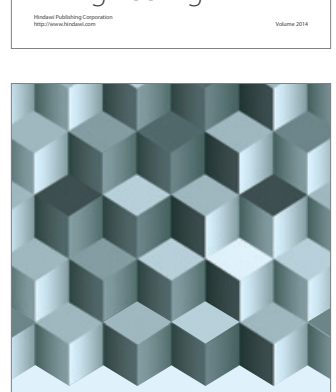

Journal of

Function Spaces
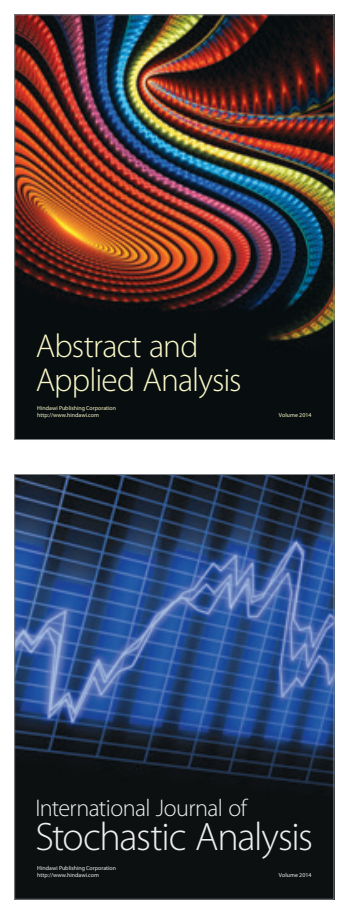

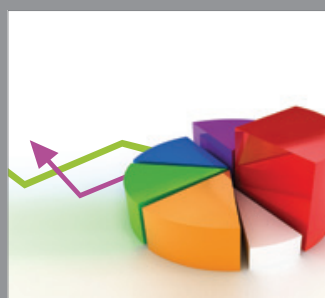

ournal of

Probability and Statistics

Promensencen
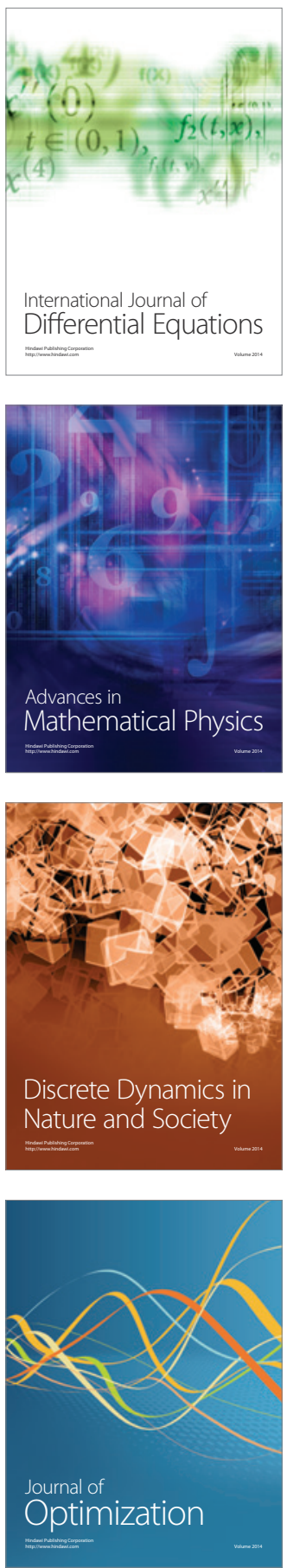